\title{
Optical Quality Control of High-Strength Structures
}

\author{
Berend Denkena $^{1, a}$, Hans-Christian Möhring ${ }^{1, b}$, \\ Wolfram Acker ${ }^{1, \mathrm{c}}$, Evgeny Zaretskiy ${ }^{1, \mathrm{~d}}$ \\ ${ }^{1}$ Institute of Production Engineering and Machine Tools (IFW), \\ Leibniz University of Hannover (LUH), An der Universität 2, \\ 30823 Garbsen, Germany \\ adenkena@ifw.uni-hannover.de, ${ }^{b}$ moehring@ifw.uni-hannover.de, \\ cacker@ifw.uni-hannover.de, 'zaretskiy@ifw.uni-hannover.de
}

Keywords: optical measurement, quality assurance, stripe projection, high-strength elements

\begin{abstract}
High-strength structure components can be built by the application of an advantageously designed geometry. These structures are challenging to manufacture due to their complex shape. This motivates the design of a new system for quality assurance of the geometric properties of these structures. Firstly, different measurement approaches and their usability for the measurement of high-strength structure components are discussed. Then an optimized solution is suggested. A pattern projection method is introduced, whereas the patterns adapt locally to the measured surface. The basic object of the research are methods of the optic three-dimensional measurement of structural elements with the help of adaptable pattern projection that allows to adjust the measuring process to the local geometry and reflecting characteristics of structural elements. This technique takes care of the special properties of high-strength structure components within the production process. It is described, how a measurement speed can be achieved that can catch up with a common production frequency without interrupting the flow of the parts. Furthermore, the measurement can take place in an industrial environment, because it is relatively tolerant to movements of the measured structure. Therefore, the measurement procedure is divided into two steps. Within the adaptation-step the luminescence and the resolution of the projected pattern is adapted to the measured object. The actual picture is taken and calculated in a second step in order to be robust against vibrations. The result of the measurement is an optimized point cloud of several million points. The paper concludes with a description of the data processing procedures necessary in order to evaluate the correctness of the measured structure.
\end{abstract}

\section{Introduction}

High-strength structure components are usually defined as parts that feature a high mechanical load capacity combined with a relatively small weight. The Collaborative Research Centre (CRC) 675, which was founded in 2006, explores new possibilities to produce and optimize those structures by controlled regulation of local properties. Most of these regulations can possibly harm geometric properties of the structure, e.g. by applying heat. Therefore, the project $\mathrm{C} 1$ within this CRC examines new methods for the quality assurance of the geometry. This is a challenging task, because high-strength structure components

- feature a complex geometry,

- are sometimes built using different materials,

- can have changing local properties,

- provide a relatively shiny surface,

Therefore, a measurement system for those structures has to fulfill high demands. It has to combine a large measurement volume with a fine resolution in designated areas of the structure. Furthermore the system has to cope with very different reflection parameters and great variations of surface angles, both properties of many high-strength structures, e.g. bar frames. In addition, the industrial environment has to be taken into account even in a research state, because of the necessity to provide a technique that is robust against vibrations and other disturbances, for example the 
influence of stray light. The following paragraphs discuss the usability of different measurement techniques for high-strength structure components.

\section{Mechanical measurement methods}

The three-dimensional measurement of surfaces is a central task of metrology in mechanical engineering. Highly established methods are mechanical approaches using either a touch probe or a continuously measuring gauge. The great advantage is precision; an accuracy of $0.1 \mu \mathrm{m}$ is state of the art in modern coordinate measuring machines [1]. In contrast to optical techniques mechanical methods hardly ever produce outliers due to the very robust approach of a physical contact between the probe and the measured surface. The mechanical measurement technique can be considered as state of the art in quality control due to their significant advantages. Nevertheless, there are several disadvantages, which are critical for the measurement of high-strength structure components. First, there is a risk of damaging either the probe or the surface due to a faulty positioning command. This is particularly significant to the measurement of high-strength structure components, because of their complex geometry. Another disadvantage of mechanical measurement is the risk of a collision of the measuring unit with the work piece. This makes the measurement of complex and unknown geometries very complicated and the automation becomes unrealizable in many cases. Such collisions can occur easily, particularly in high-strength structures, because the surface has many holes to be measured. Furthermore, mechanical measurement techniques yield comparatively few points and therefore they take a long time to measure a complex surface. Hence, the measuring time makes a real-time inline quality control impossible.

\section{Optical measurement methods}

Optical measurement techniques have gained significance in production engineering within the recent years. Their advantages are a measuring speed that is higher by decades compared to mechanical approaches and the possibility to measure contact-free. Hence, the risk to damage either the probe or the surface is eliminated. Due to the various applications of form measurement, a wide variety of different optical measuring approaches has been developed. They can be divided into several categories. One possibility is to distinguish the form of light used, whether coherent or not. Coherent techniques are e.g. holography, shearographie and the white light interferometry [2]. Because interferometric approaches use the interference effect for measurement, it is usually necessary to position the object very stiffly to guarantee a clear phase signal. This makes it difficult to use such systems in rough environments. Furthermore, the measurement volume is usually very limited. Therefore, these approaches are not useable for the described aim. An exception is the shearographie, but this technique is only able to measure differences between two measurements and therefore not suitable for form measurement.

Non-coherent methods also utilize laser light to some extent, but it is not used here because of its coherence. These methods often operate on the principle of triangulation (Fig. 1). The depth information of a camera image is interpreted as a laterally directed shift due to a side light. Although all triangulation methods are based on this basic principle, there is a variety of different realization forms, such as shading methods, photogrammetry and measurement using structured illumination. The shading method attempts to calculate the surface due to the brightness of the individual image points. However, this is only possible for objects with a very favourable reflective, well-known and constant surface structure that cannot be assumed in high-strength structures. Furthermore, this method also has a high measurement uncertainty. The photogrammetry and measurement using structured illumination are described in the next paragraph.

\section{Photogrammetric and Pattern Projection Approaches}

In photogrammetry, two cameras are used to observe the surface from two different perspectives. An algorithm identifies significant corresponding points of the surface in both camera pictures. The 
height of this point can then be calculated from the offset between the representations of this point within the two camera pictures at a given angle between the cameras. This effect is used in nature as well as an aspect of our three dimensional perception of the sight. A prominent problem of this approach is the need of a sufficiently textured surface in order to find corresponding points in both camera pictures. As many technical surfaces don't feature such a surface structure, a structure can be applied to the surface by projecting a pattern onto it. This pattern is typically a stripe pattern, which yields a very good contrast in transition. But the method is dependent on the use of at least two cameras. The measured points have to be clearly visible in both pictures, which significantly lowers the range of measurable surface angles. Principally a second camera is not needed if enough knowledge about the projected pattern (e.g. stripes) exists. This method is used in so called "single eye" measurement approaches. The projection device can be a conventional laser, a line laser or a projector. If the projection device is a laser line, the approach is called light segmentation. The result of the analysis of a single picture is a vertical section of the measured object. Thus the object or the measuring line has to be moved and different measurements have to be combined in order to obtain a dense point cloud of the object's surface. The principle of all these methods is to use the difference of perspectives of camera and projector. The angle between camera and projector is called "triangulation angle". The projected pattern is deformed depending on this angle and the height of the measured object.

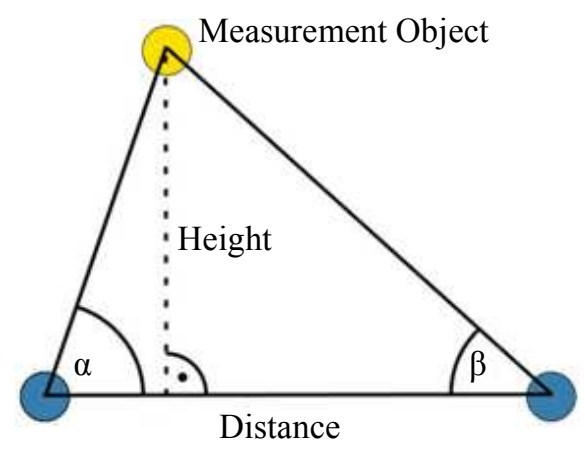

Fig. 1: Height measurement with triangulation

Fig. 2 shows a simulated projection of a regular stripe pattern onto the surface of a sphere. The camera looks at the sphere from an angular position. In the left part of the picture the stripes are projected horizontally. Obviously the stripes are not deformed significantly, because information within the projected pattern only varies normal to the plane set up by sphere, projector and camera. In the right part of the picture the stripes are projected vertically, thus producing a maximal signal. Other patterns e.g. stochastically generated patterns are used as well.

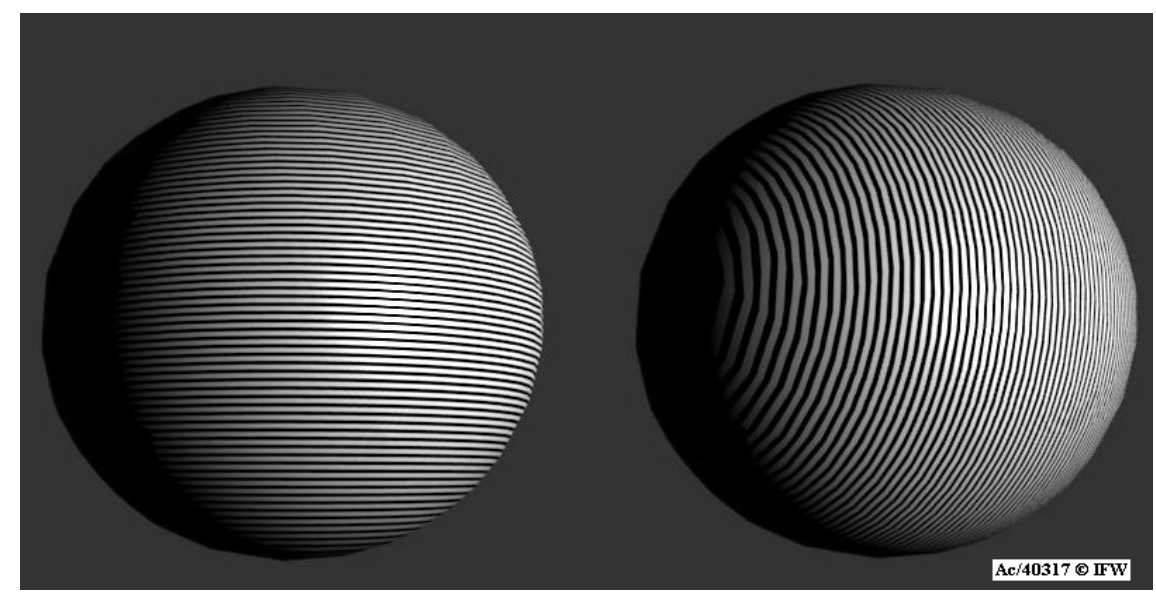

Fig. 2: A regular stripe pattern, projected onto the surface of a sphere 
However a significant problem that remains is to distinguish the projected stripes due to their periodicity. This problem can be solved with a coded sequence of projected patterns that generates a unique identifier for each camera pixel either by projecting a binary (usually gray-coded) sequence of stripe patterns [6] or two patterns with slightly different frequencies in order to get an identifier by the beat.

The gray-coded sequence of stripe patterns is illustrated in Fig. 3. The individual light planes are indexed by an encoding scheme for the light patterns. These light patterns lead to a unique code for every plane and reduce ambiguity problems.

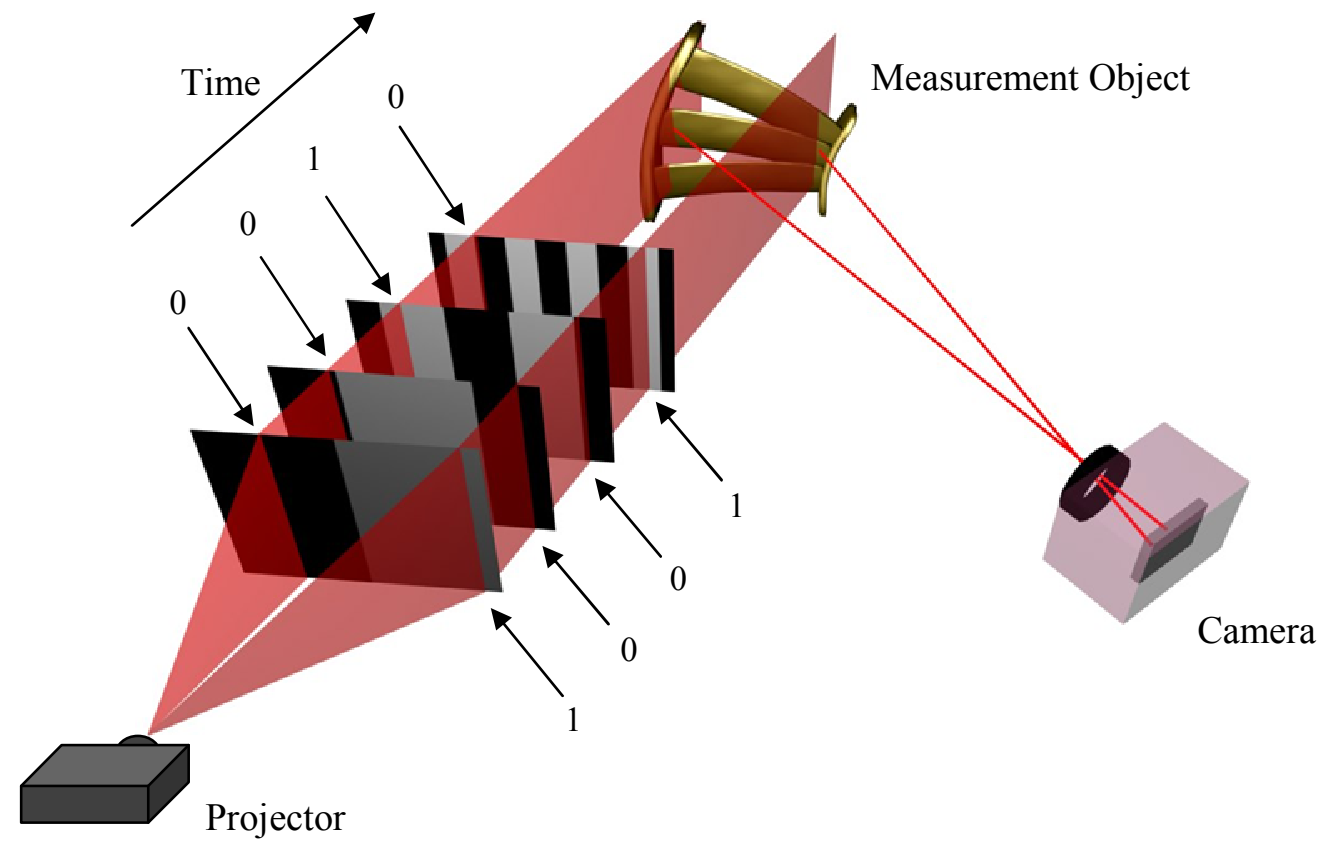

Fig. 3: Gray-coded sequence of stripe patterns

The disadvantage of light stripe projection is that the light stripes can be determined only discretely. Hence, the measurement resolution is limited to the selected bit resolution. With the help of continuous functions, such as a sine wave, the measurement can be continuously resolved. This allows not only to interpret the light/dark edge, but to make a whole phase-analysis $[4,5]$. Thereby it becomes possible to measure a denser cloud and to provide a sub-pixel resolution of the depth information.

The correspondence problem due to the periodic character of the sine function is fortunately dissolved in combination with a light stripe projection. The idea of this method is that the periodic stripe pattern is shifted $n$ times by the phase $2 \pi / n$ (Fig. 4). To determine the phase with $n$ steps shifting the following formula is given [10]:

$$
\tan \varphi=-\frac{\sum_{i=0}^{n-1} I_{i} \cdot \sin \left(\frac{2 \pi}{n} i\right)}{\sum_{i=0}^{n-1} I_{i} \cdot \cos \left(\frac{2 \pi}{n} i\right)}
$$




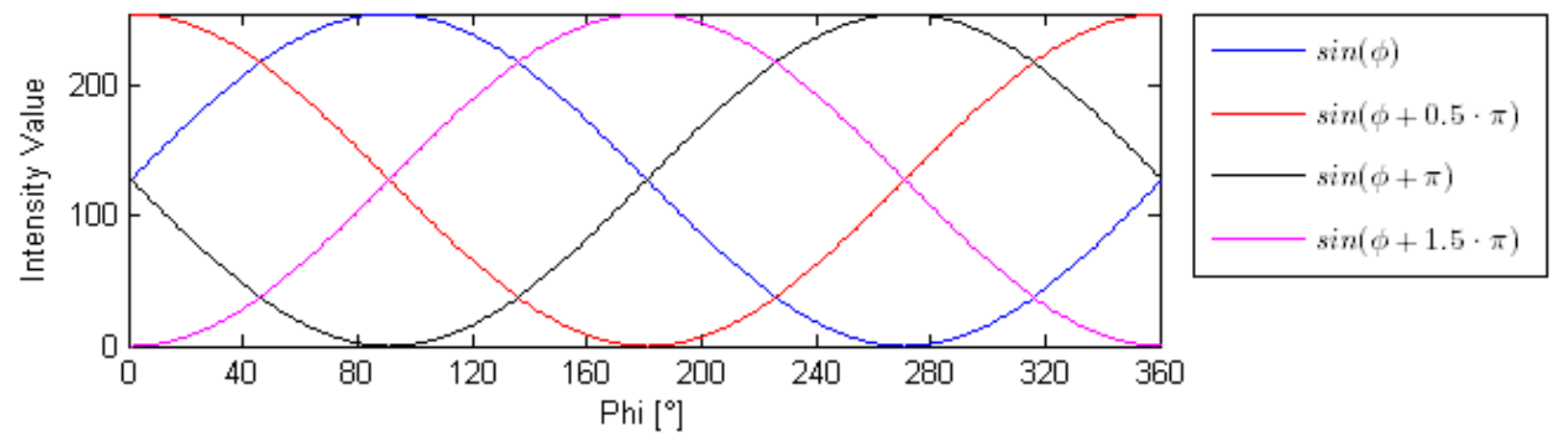

Fig. 4: Phase shifting implemented with sine wave

There are required at least $n=3$ shifts. In this work a four-phase shift has been applied. Based on the equation (1.1) follows:

$$
\tan \varphi=-\frac{I_{3}\left(\frac{3 \pi}{2}\right)-I_{1}\left(\frac{\pi}{2}\right)}{I_{2}(\pi)-I_{0}(0)}
$$

The values $I_{0}, \ldots, I_{3}$ are the observed intensity values for the considered pixels.

The problematic part of these techniques is that multiple pictures have to be taken and to be interpreted cohesively. If the measured part moves slightly during the measurement, for example due to vibrations, the accuracy of the procedure can drop dramatically, because the over-all accuracy deteriorates if the algorithm confuses the projected patterns. Therefore, a new approach is examined here using a sequence of pictures in order to pre-measure the surface followed by a single measured picture with locally optimized illumination and adapted resolution.

\section{Two-Step Measurement}

An important goal of the project is to gain a more robust measurement procedure and to accelerate the necessary data processing of the measurement. In the last paragraph the disadvantages of an approach with a coded sequence and a single-picture approach were discussed. This leads to the idea of combining both strategies using the best properties of both techniques.

Complex surfaces require a high resolution of the point cloud in order to accurately record important features e.g. the position of edges. This was only possible with a high-resolution total measurement, which provides a relative large point cloud. The complex image processing algorithms that are used for quality control, take a lot of computing time, because all points of the point cloud have to be analyzed. If the density of the cloud of points could locally be reduced in a way that at the same time the quality control does not get affected, the execution of the algorithms used in image processing could be accelerated by a considerable degree. This means to be one step closer to the real-time capable quality control.

In order to adjust the resolution of the actual measurement of the component locally, an adaptive approach has been investigated. The procedure is divided into two major steps, the "Adaptation step" and the "Measurement and Evaluation step". Within the "Adaptation step" a prior knowledge about the geometry is generated and the projected patterns are changed until an optimal picture for the measurement task is found. In addition, the critical areas are identified and the resolution of the pattern at these points is locally determined. The resolution of the projected patterns is adapted locally to the surface. If the surface yields less information in one region, e.g. a plane or a cylinder, the resolution of the projected pattern is reduced, while complex formed sections are scanned at the maximal possible resolution. This enhances the informational content of the finally measured point 
cloud and the robustness of the procedure, due to the fact that the bigger patterns used to measure the less complex parts of the structure yield more robust depth detection.

Furthermore, additional information about the spots of complexity on the surface is generated, which helps referencing the coordinate systems of the measurement and the model of the geometry. Pattern and luminance adaptation require information about the geometry before the adaptation can take place. Therefore, a pre-measurement is done as a first step within the "Adaptation Step". This measurement uses a robust sequence projection approach with very limited resolution in order to create the information necessary. Fig. 5 shows the measurement strategy of a structure with local high-resolved model at the joint.
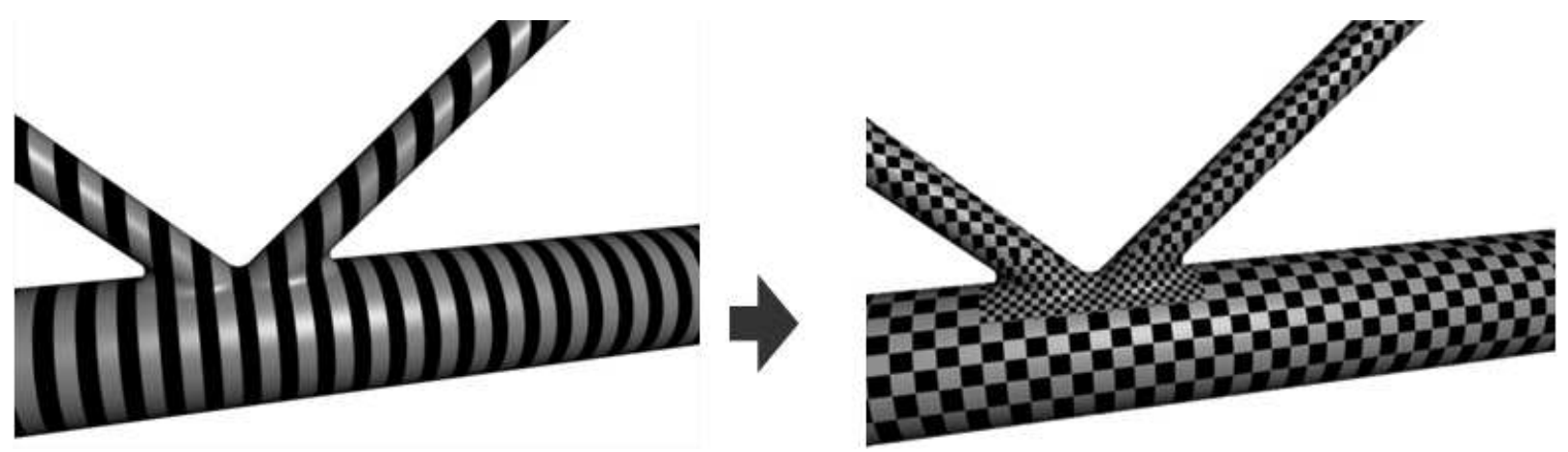

Fig. 5: Measurement strategy with local resolution

The luminance is also adapted locally to the surface of the measured structure. This enhances the robustness against complex surface angles and problematic reflection parameters by reducing the signal to noise ratio in the camera's signal. The luminance adaptation will be described in detail in the next paragraph. Fig. 6 and 7 illustrate a measurement procedure. A simulation of a possible procedure can be seen in Fig. 19.

After the projection is adapted, the actual measurement takes place using a single picture [11]. The result of the measurement step is a point cloud. The next task is to generate information about deformations and the quality of certain features of the measured part using the point cloud. This step is a very complicated procedure, which takes a lot of calculation time. The most time consuming task is to transform the coordinate system of the structure into the coordinate system of a reference model, which can e.g. be a CAD model of the structure. However, there are some cases in which a CAD model of the structure either does not exist, or does not resemble the measured geometry sufficiently to gain information for quality control. In this case it can be reasonable to compare the point cloud with a cloud of a prior measurement of a not faulty part.

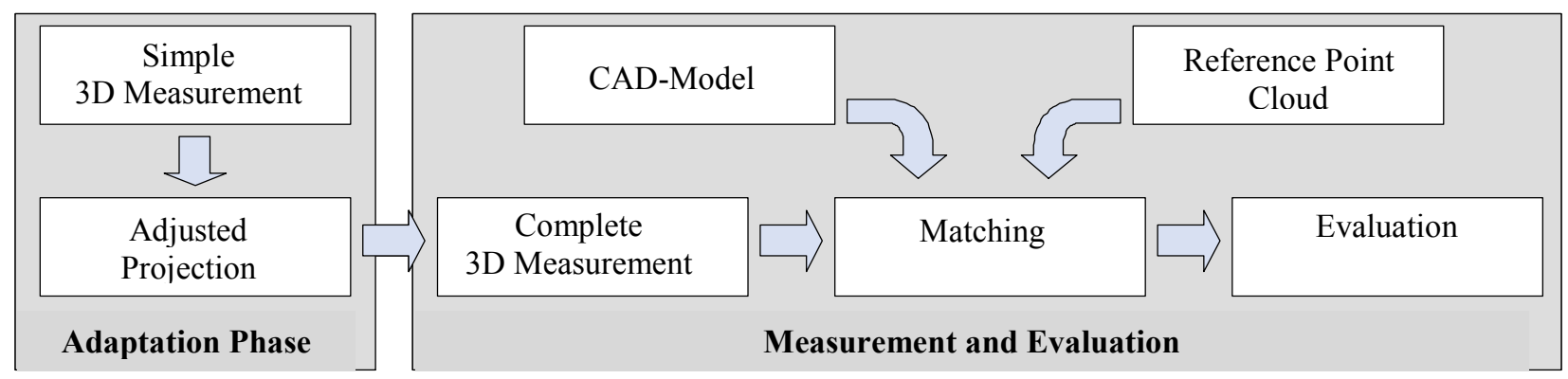

Fig. 6: Measurement principle 


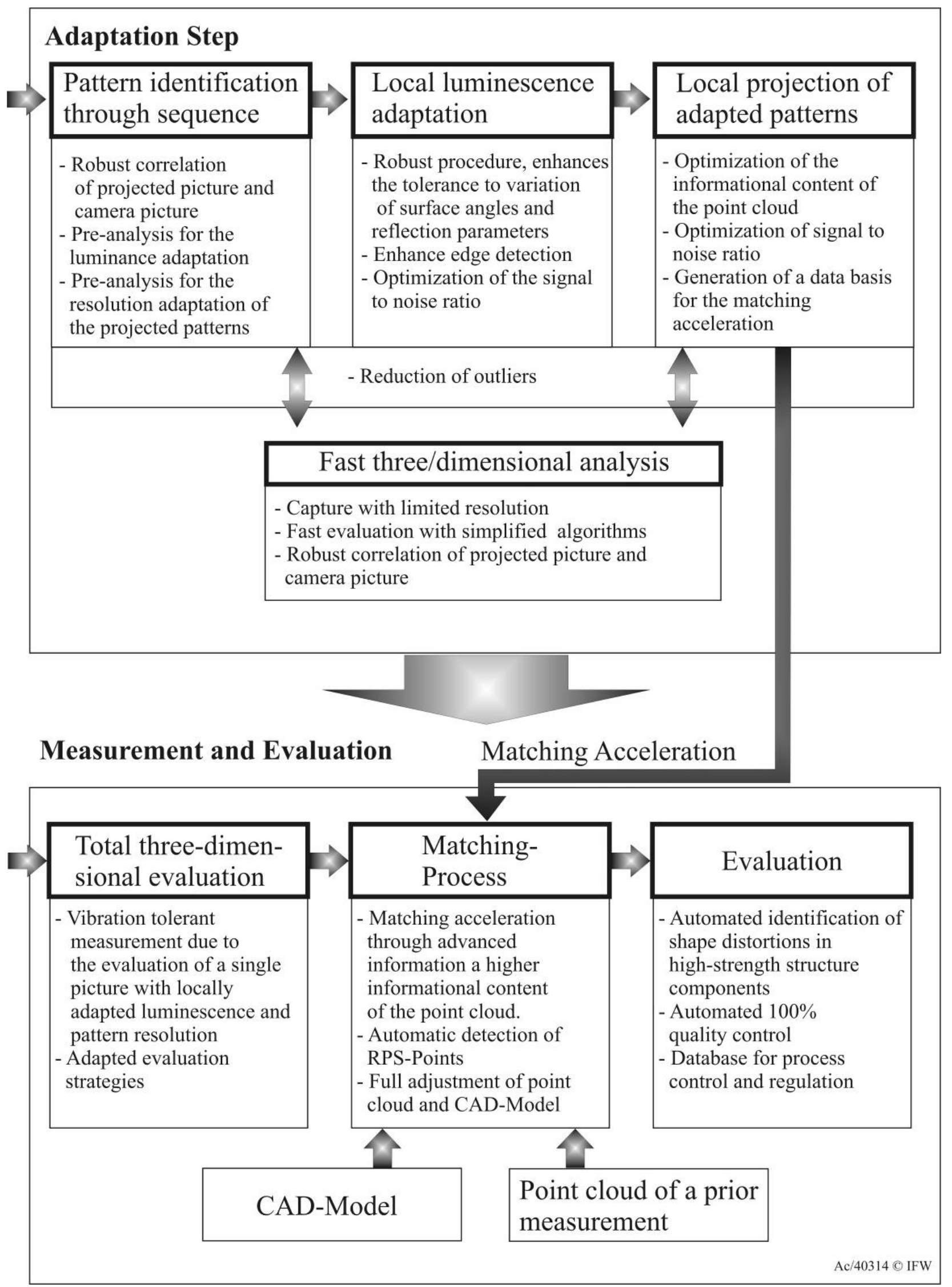

Fig. 7: Two step measurement process 


\section{Luminance Adaptation}

The accuracy and performance of recent form measurement setups is significantly limited by the reflection parameters of the measured object [7], whereby a diffuse reflecting white surface can be considered as the optimum. Problematic structures are usually prepared prior to the measurement e.g. by applying a layer of titanium oxide. This preparation as well as the necessary cleaning after the measurement procedure is contradictory to the aim of a $100 \%$ measurement within the production chain and the layer limits the measuring accuracy by its thickness. Furthermore, highstrength structure components often feature particularly difficult reflection parameters, because they often provide metallic, shiny, complex-formed surfaces with an inhomogeneous texture. These factors lead to a wide variety of intensities in the camera picture. The limits due to reflection therefore have to be exceeded in order to measure high-strength structure components. Until now, the only adjustment of the intensity is the general exposure time of the camera. The possibility of using the projected image has not been used yet. Therefore, a local luminance adaptation of the projected pattern will be implemented within this project. The problem is the necessity to know which part of the camera picture, which is e.g. too bright, is represented by which part of the projection picture. Strictly spoken, it is exactly that information that is to be measured in order to gain the three dimensional information. Thus, a first rough measurement is run in order to get a simple model of the geometry. This can be a very low resulted gray pattern. Another even more robust method is to project a very unique pattern (e.g. cross) in multiple pictures onto the entire surface of the measured object. Then, the projected picture is divided into patches of the size of the pattern. By applying three dimensional algorithms on the two dimensional surface, the angle of each patch can be calculated relatively exact. The luminance of each pad is then modified in order to achieve an optimal illumination. The luminance can also be modified in the sub-patch spaces by moving the centre of illumination within each patch.

\section{Intelligent Matching}

The task of identifying objects within pictures and measure features is accepted as a very complex and demanding task. The generation of measurement data from a point cloud is even more demanding. For quality control purposes there are three different approaches of interpreting the measured data in order to evaluate the quality of the structure. The first is to compare the data with a reference measurement; the second is to compare the point cloud with a model, e.g. a CAD model. A third way would be to directly evaluate certain features, like planarity. In each case it is important to pay attention to the fact that the measured data and the model (CAD or mathematical principle) are not within the same coordinate system. It is therefore necessary to acquire a transformation between the coordinate systems. This step is not trivial and is often performed using a variation of the ICP (Iterative Closest Point) algorithm [8,9].

At first, for every point of the measured cloud a so called "neighbour" is calculated. This can either be the nearest point of a second cloud, or a surface point of a CAD-model. Then the best possible transformation between the point cloud and the neighbour-cloud is calculated. The transformation is then performed and the algorithm is started again (iterative). It can either be stopped after a fixed count of iterations or if a given error rate is fallen short of. The method has two main disadvantages which currently disqualify it as a standalone solution for a full quality control. The first is simply that it really detects a very good solution for fitting both systems. If the measured geometry is faulty, the algorithm still calculates a condition in which both models will fit best. This can lead to an underestimated fault and wrongly detected faults. Fig. 8 illustrates this problem using a two-dimensional example. To overcome this obstacle so called RPS-points (reference point system) can be defined within the measured part. The centre of a drill is an example for such a point. The RPS-points can be detected automatically within the measured geometry after the global position has been found using the described ICP-algorithm. The remaining transformation can then be performed by using the RPS-points. 

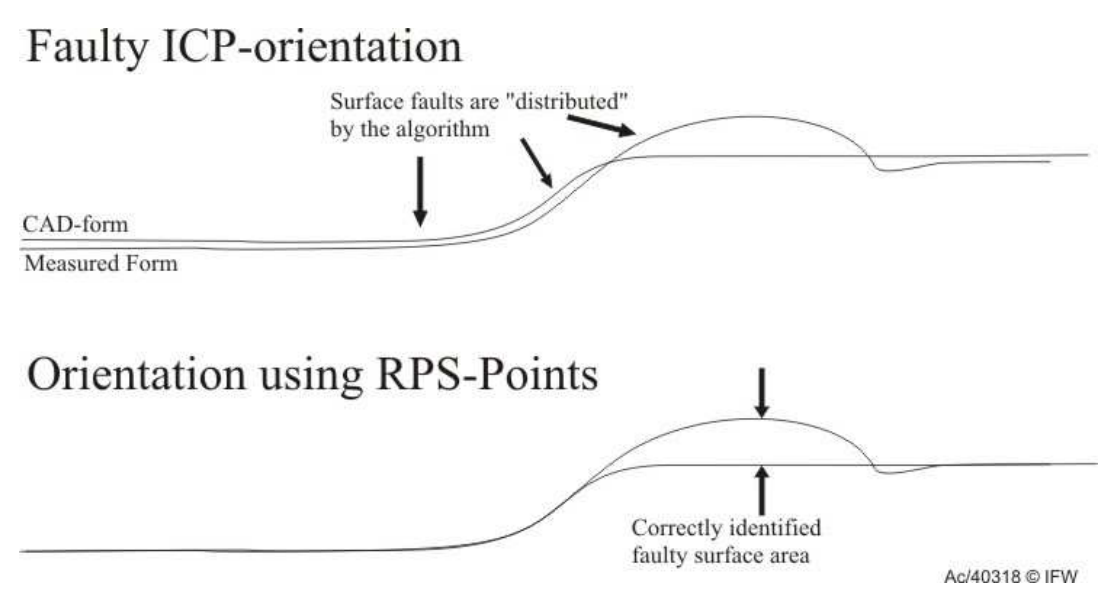

Fig. 8: RPS-Matching

The second disadvantage of the ICP-method is the calculation time needed by the algorithm. Due to the fact that a corresponding point has to be calculated for each point of the cloud, the calculation time of an optically measured cloud - which can consist of several million points - does not allow a real-time measurement of all produced parts. Therefore, a technique is being researched to reduce the number of points in less significant parts of the structure within the measurement process. This method can also used to generate additional information in order to speed up the matching process. The matching process is slowed down significantly by two factors. Firstly, the calculation time rises more than quadratic with the size of the point cloud. Secondly, the algorithm is notably decelerated by the number of outliers. This can easily be understood by the fact that a group of outliers provide a very far away geometry, which statistically produce a relatively big part of the transformation leading into the wrong direction, thus retarding the iterative movement of the cloud and obviously limiting the quality of the result. A general idea to overcome both problems would be to simply decrease the resolution of the measurement. But this would mean to e.g. "jump" over an edge hence destroying the information needed to generate RPS-points.

The solution suggested by this paper is a resolution adaptation during the measurement process. The necessity to measure within a single "shoot", which means to generate a resolution adapted pattern in order to realize resolution adaptation, has to be kept in mind. After that, a single high resolution camera picture is used to calculate the point cloud. This technique yields another advantage. Due to the bigger patterns in less structured parts of the object, the reliability of those points is increased significantly thus reducing outliers. This can be taken into account during the matching process, by applying a "weight" to every point of the cloud. Points measured with a bigger pattern can be considered more reliable, thus getting a higher weight and speeding up the process.

\section{Implementation of algorithms for distributed image processing}

The increasing demands on product quality are associated with major influences on the manufacturing accuracy. The form-error detection and measurement systems on the market are too slow for the increased production cycle. The previously known image processing algorithms are programmed only sequentially and the usage of increasingly complex data results in more computing time to process image data. The goal is to make measurements and comparisons in the flowing production in order to achieve the real-time capability. Therefore, the computational intensive image processing algorithms have to be parallelized to raise the processing speed of data. The study examines algorithms for image processing adjusted with the help of modern techniques for parallel operation. By this method the computing time is shortened by the use of multi-core processors or a high performance computer. Thereby the ability to deal with increasing computational complexity will be achieved. Particularly high computational processing time arises in the implementation of a comparison between measurement and a reference model. In this report, 
the strategy of distributed computing is discussed using the example of so-called ICP (Iterative Closest Point) algorithm.

The measurement was performed on a cluster computer. The cluster consists of eleven nodes each with two Intel Xeon 5405 quad-core processors and 2GB of system memory. The 64-bit version of Windows Compute Cluster Server 2003 is used as an operating system. The nodes are connected with each other using two separate Gigabit Ethernet networks (Fig. 9). One is used as an MPI (Message Passing Interface) network and the other as a general data network. The program is set up in a client - server architecture to allow the separation between the MPI capable program and a graphical user interface. The measurement computer could use the computing power of the computer cluster, thanks to a network connection. One single instance of the server program, which is known as the master process, runs on the main computer of the cluster. The other application instances are running on client machines through MPI and are referred to the slave processes. The master process acts as a coordinator and is involved in communicating with the client program that distributes the workload evenly to the slave processes to the calculation results are then sent back to the client.

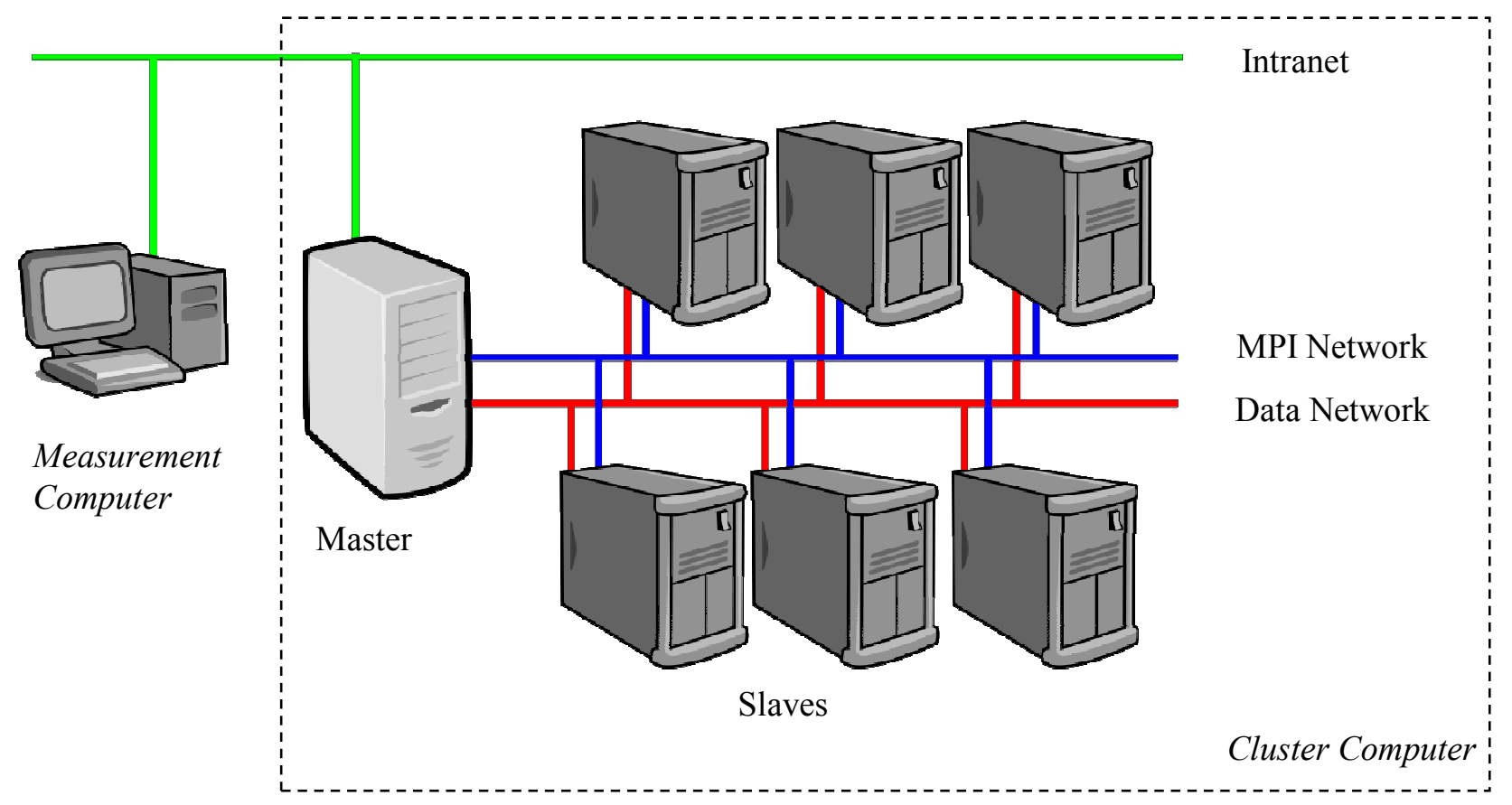

Fig. 9: Cluster computing, network technology

After the process of the shape acquisition the created point cloud will be compared with the CAD model. There is a problem that the two point clouds are not in the same coordinate system. The ICP algorithm has the objective to provide a transform which maps the points of the first coordinate of the second in three-dimensional space. Fig. 10 shows the execution graph of the ICP algorithm. 


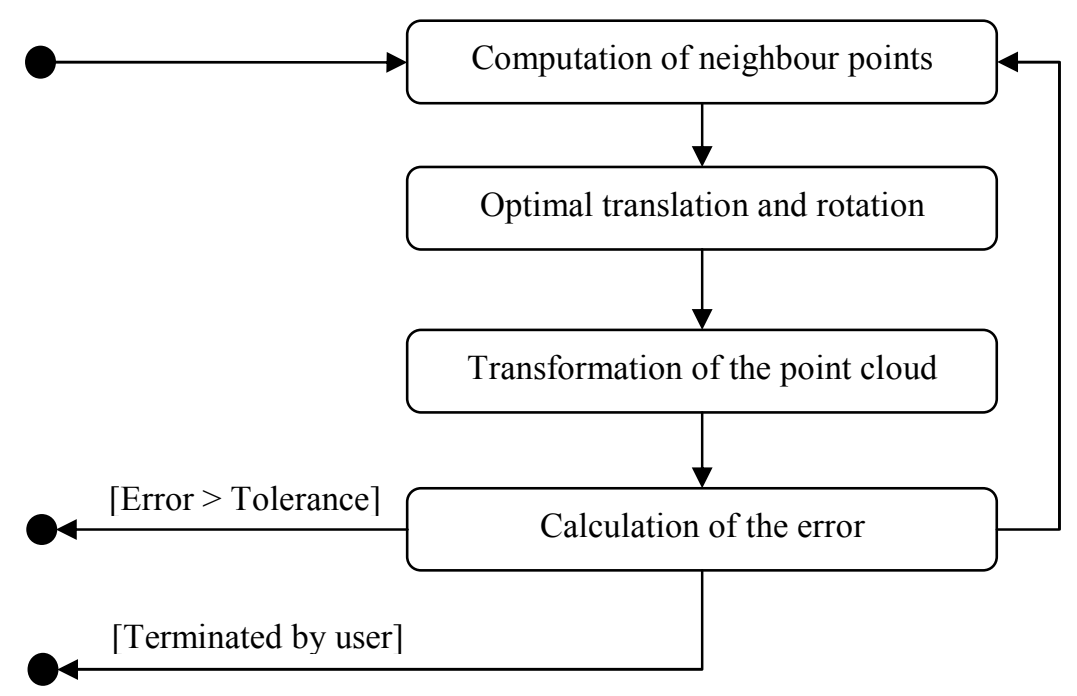

[Else]

Fig. 10: ICP algorithm

The scalability investigation of the ICP algorithm was tested according to the following approach. Eight point clouds were composed of a varying number of points. The smallest cloud consists of 255 points and the largest of more than 1.16 million points. Due to extremely high processing time of clouds with over 100,000 points, the first five clouds were tested with all possible number of kernels. The tests with the last three point clouds have been carried out only at 16, 32, 48, 64 and 80 cores. The point clouds were generated from STL files through the client program and then sent to computing clusters for processing the parallelized version of the ICP algorithm. Since the algorithm consists of several iterations, which would equal last, only the time duration of the first iteration has been measured. The timing is started directly after the point cloud file was sent by client program and stopped after the receipt of the index table and the error value.
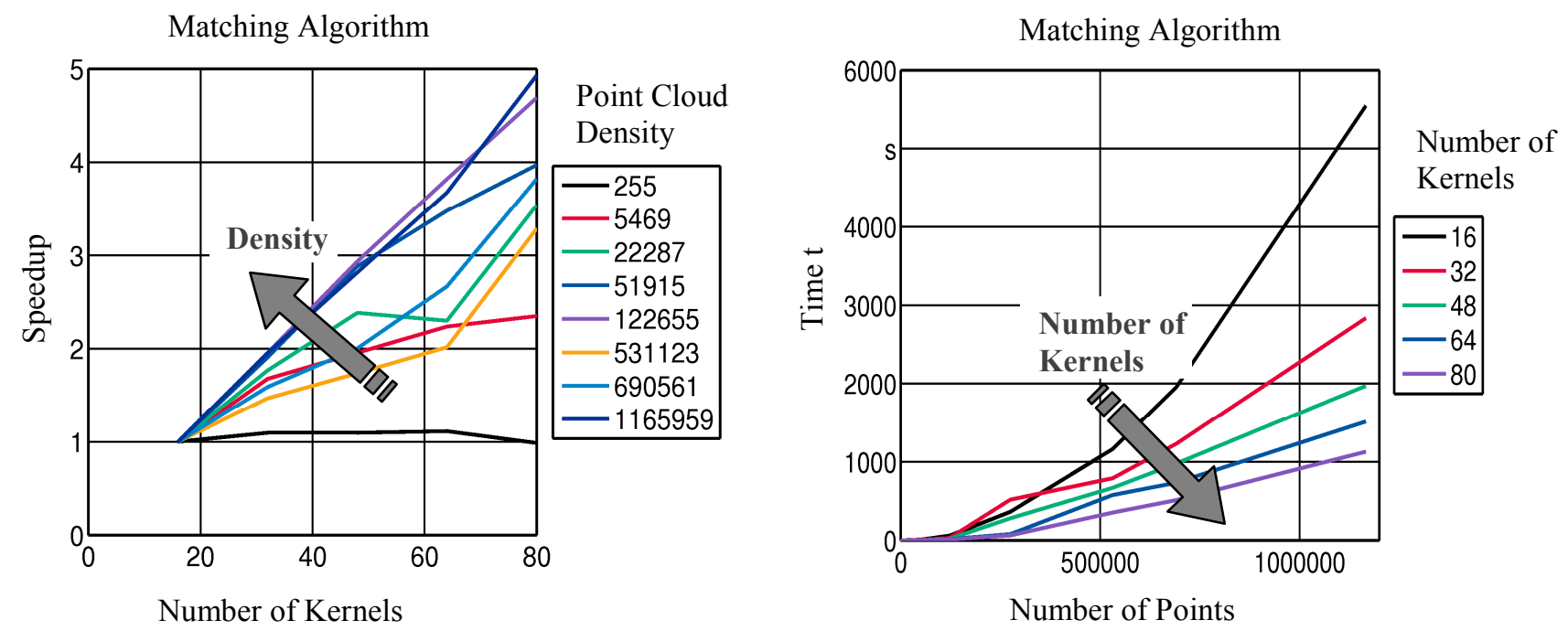

Fig. 11: Timing results of speedup 


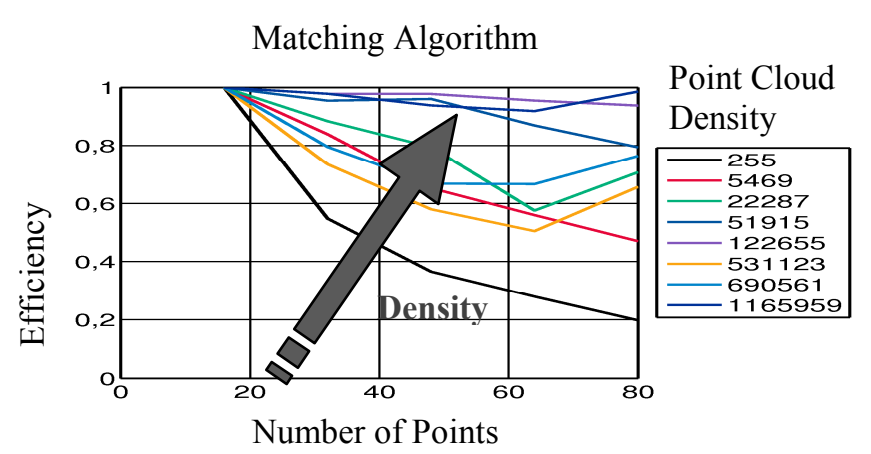

Fig. 12: Timing results of efficiency

The parallelized version of the ICP algorithm offers an excellent scalability with an efficiency value of about 0.8 (Fig. 12). However, for real-time capability of the algorithm for point clouds with over 50 thousand points the true high-performance computers are needed.

\section{Experimental Setup}

The strategies developed are investigated using the experimental setup shown in Fig. 13. As a projector a video projector with a high intensity of light is used. The base is constructed very stiffly in order to minimize movements of the projector, the camera and the measured object. The camera can be moved in various angles in order to investigate the influence of the triangulation angle.

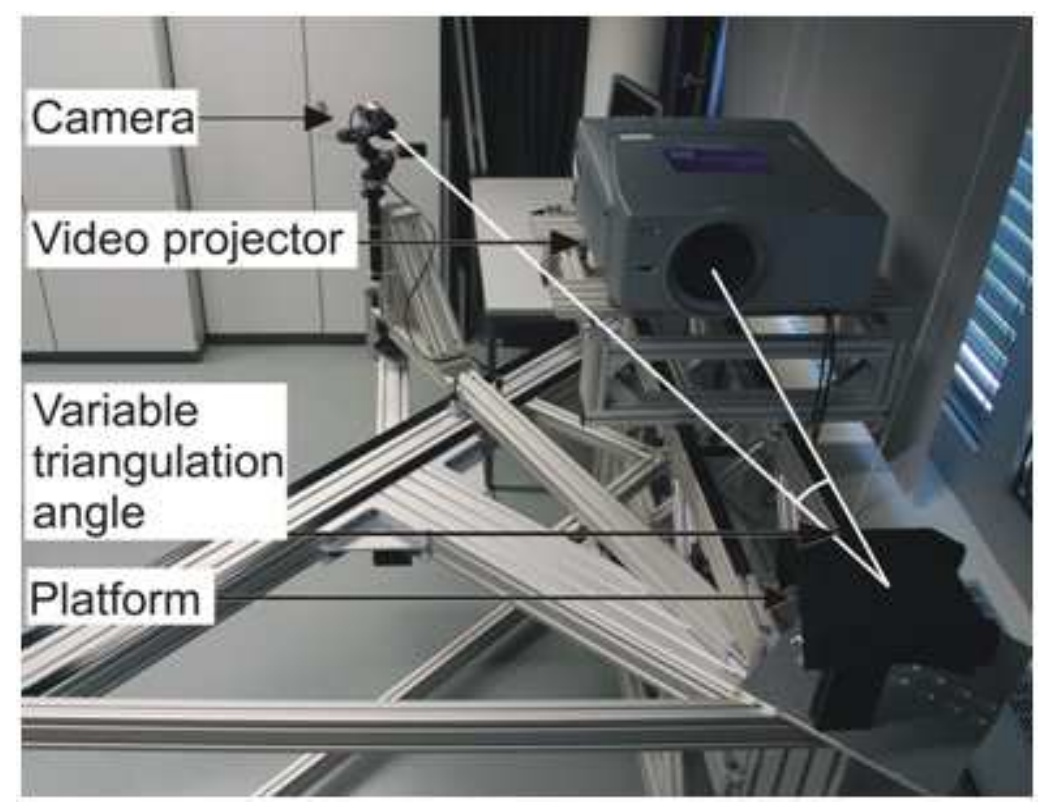

Measurement Object Size

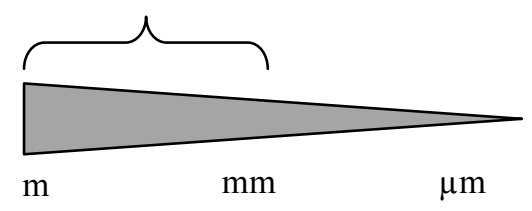

Fig. 13: Measurement setup (macro-system)

The depth resolution of the measurement setup shown in Fig. 13 is up to $0.2 \mathrm{~mm}$. In order to analyze also smaller structures, a micro-measurement system was built additionally with the aid of a microscope. The same measurement strategy is applied also in this system. The new feature gained here is a higher measurement resolution by coevally smaller object size. This micro-measurement setup is shown in Fig. 14. The depth resolution with this system rises up to $1 \mu \mathrm{m}$. 

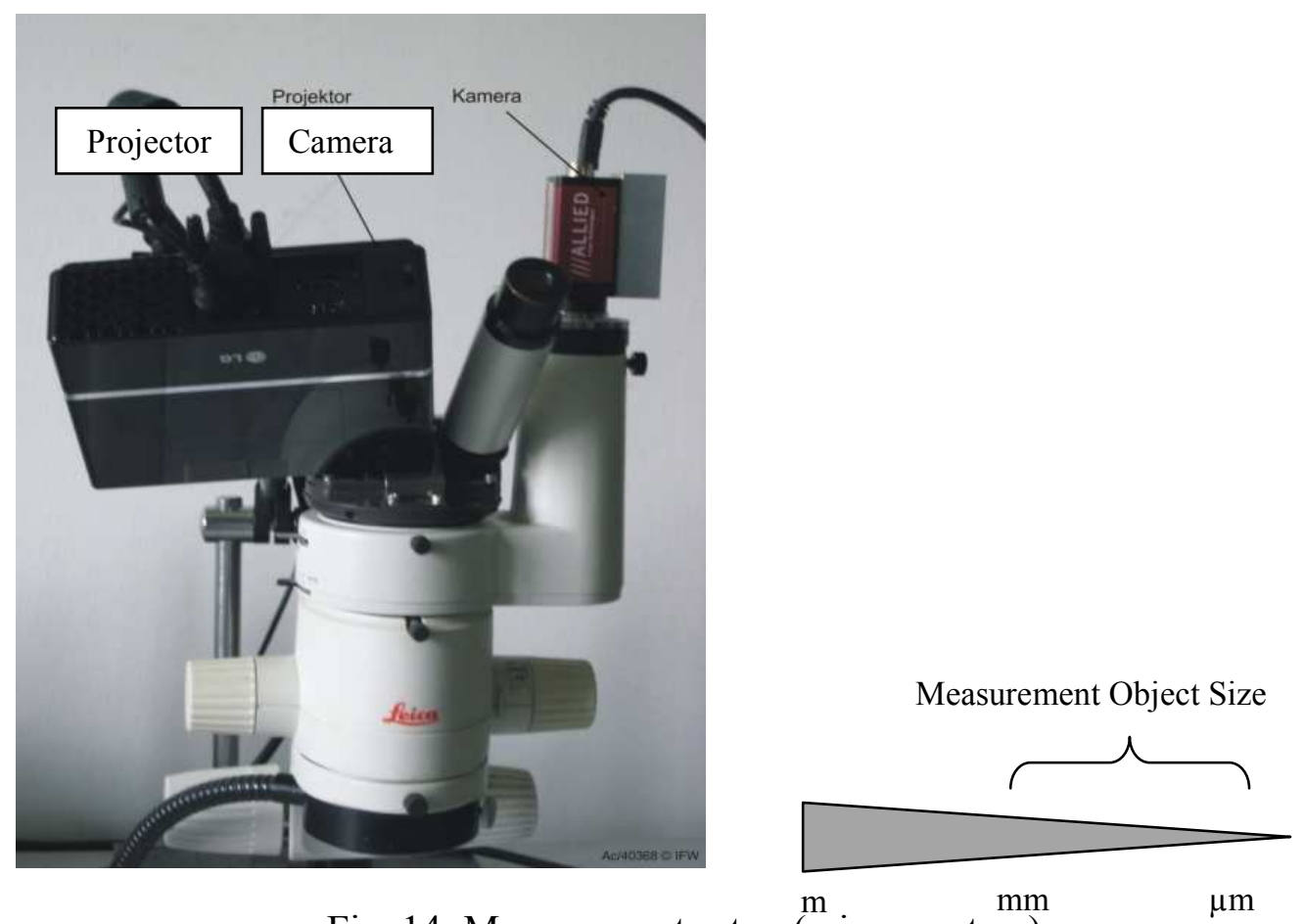

Fig. 14: Measurement setup (micro-system)

\section{Measurement results}

Some measurement results of an exemplary high-strength structure component are presented in Fig. 15.
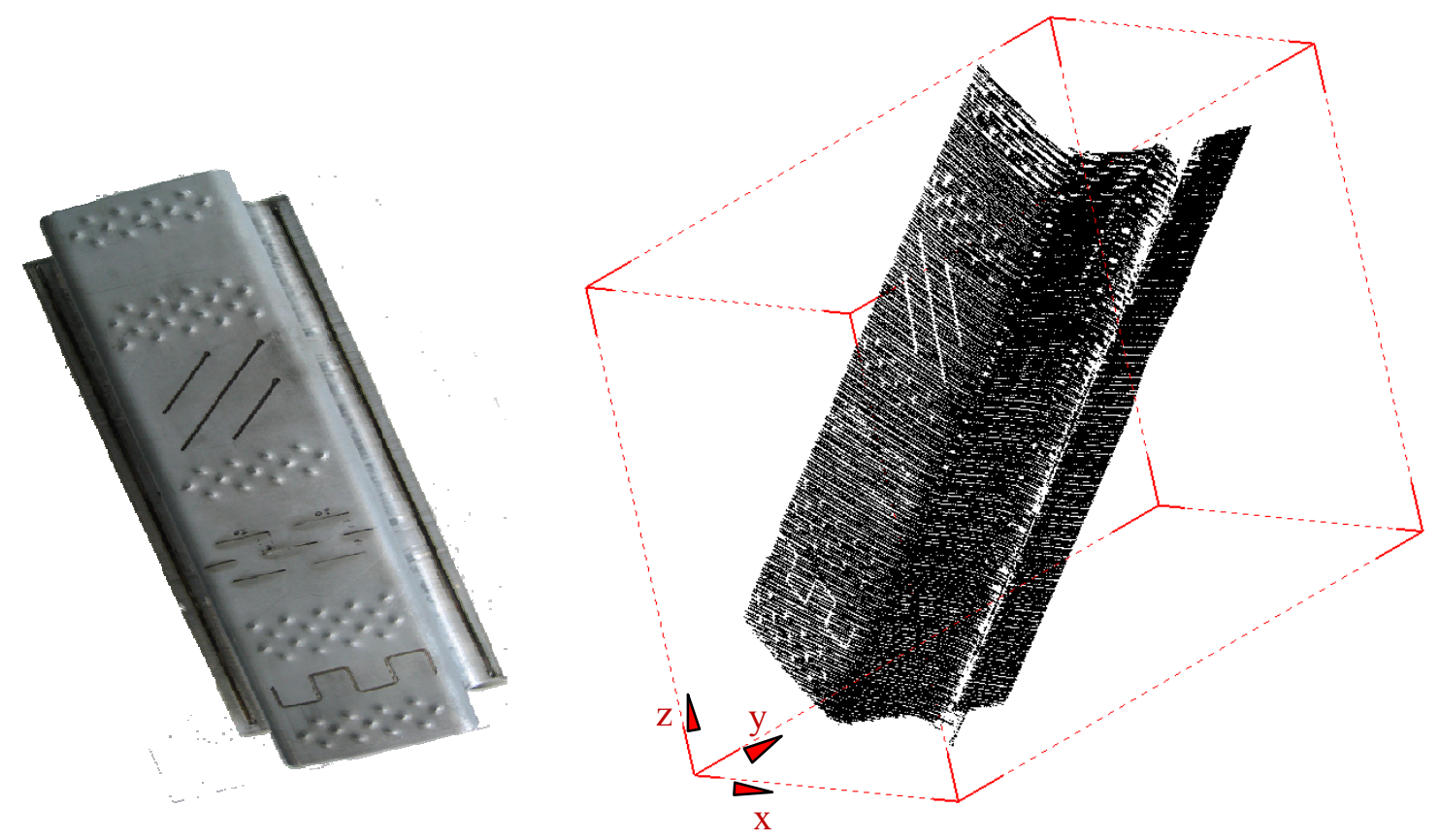

Fig. 15: Measurement object and corresponding point cloud

In order to verify the measurement accuracy of the optical measurement the matching and difference analysis with the point cloud of the tactile measurement was performed. The both point clouds with color depth information are displayed in Fig. 16. The difference analysis shows results for the deviation error of $0.3 \mathrm{~mm}$. 


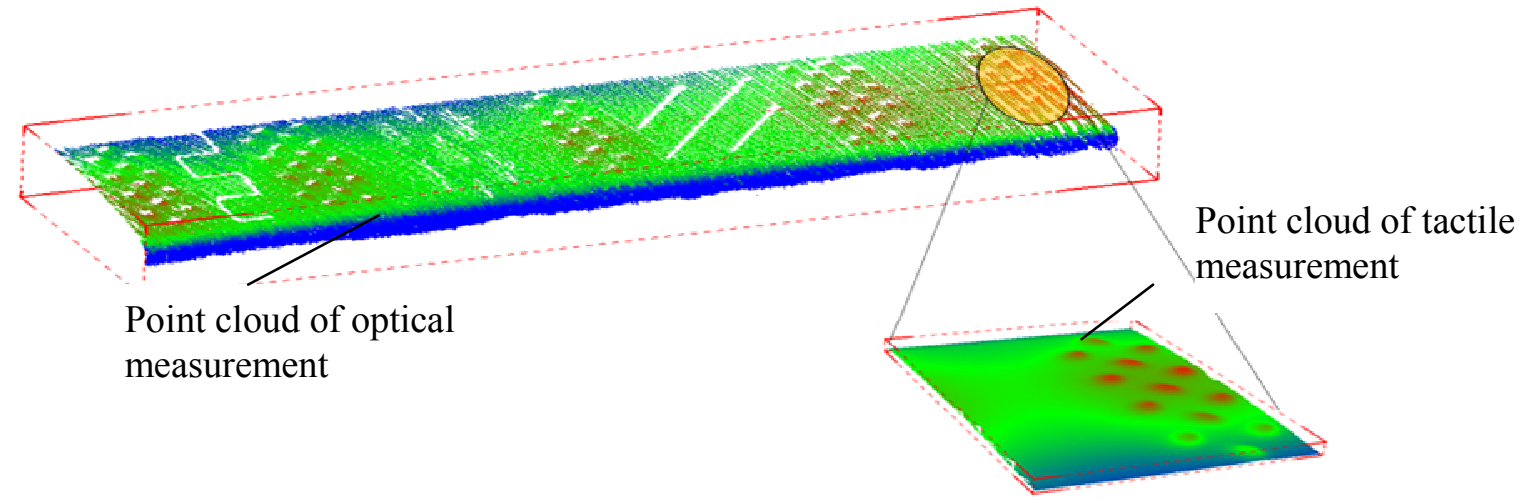

Fig. 16: Comparison between optically and tactilely measured object.
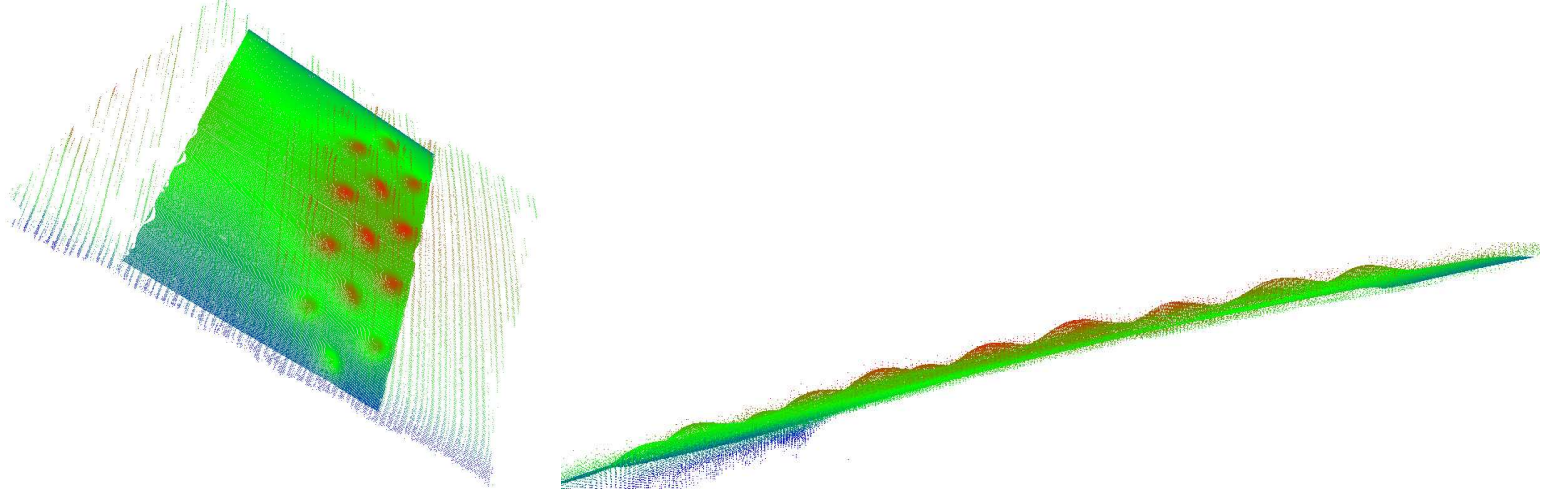

Fig. 17: Matched point clouds shown from different perspectives

\section{Simulative testing of the used strategies}

The strategy pursued here for the high-resolution detection of high-strength structures is based on a two-step shape measurement method. First of all, a rough shape measurement is performed, which allows identification of critical points on the component and the locally outshone areas. An adjustment of the local luminance and the resolution of the pattern are performed based on these data. In the second step, the component is measured in high resolution. For the simulation of this strategy, a ray tracer was developed.

The described strategies can be tested in the simulation with every possible material, adjustable camera and projector resolution and several disturbing light sources. Fig. 19 provides an impression and visualizes the measurement process. Furthermore simulations are used for the implementation of the luminance adjustment. The ray tracer visualizes also various models for the reflection of light on technical surfaces. The reflection model and its parameters are then used to predict the weakening of the light due to the surfaces angles und properties within the measurement.

The measurement cycle with a simple striped pattern is shown in Fig.18. The luminance and the pattern adjustment gained from the first measurement information are implemented in the actual measurement. The adjusted projection allows a more homogeneous and therefore stable measured value acquisition.

a)

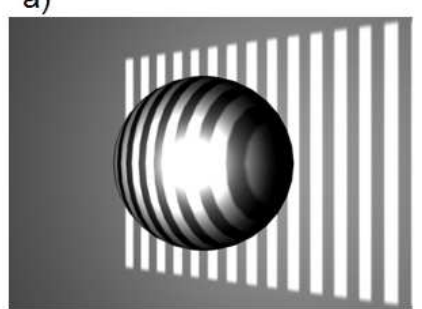

b)

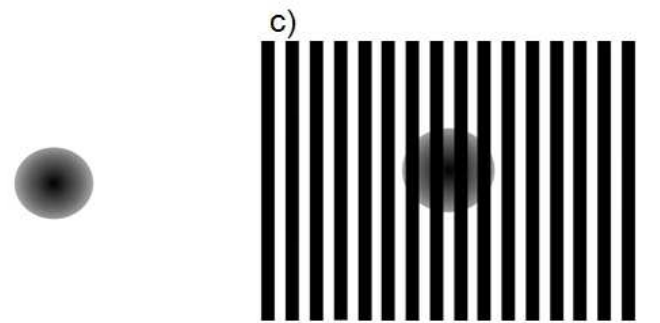

d)

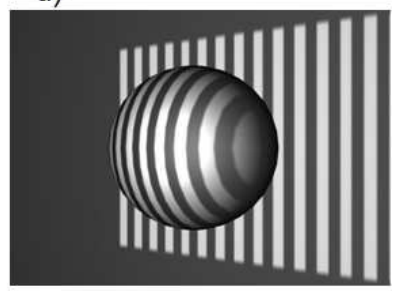

Fig. 18: Strategy of luminance adjustment a) Adaptation phase; first measurement b) Calculation of the contrast mask c) Adaptation of the pattern d) Luminance adjusted measurement 
First, the gray code method for the optical measurement of components is used. In the second step, a sine wave pattern is projected onto the component and gradually shifted so that a phase-sensitive measurement could be performed. By phase-shifting a high-resolution determination of components is possible. But it needs the Gray code method to make an assignment unambiguous.
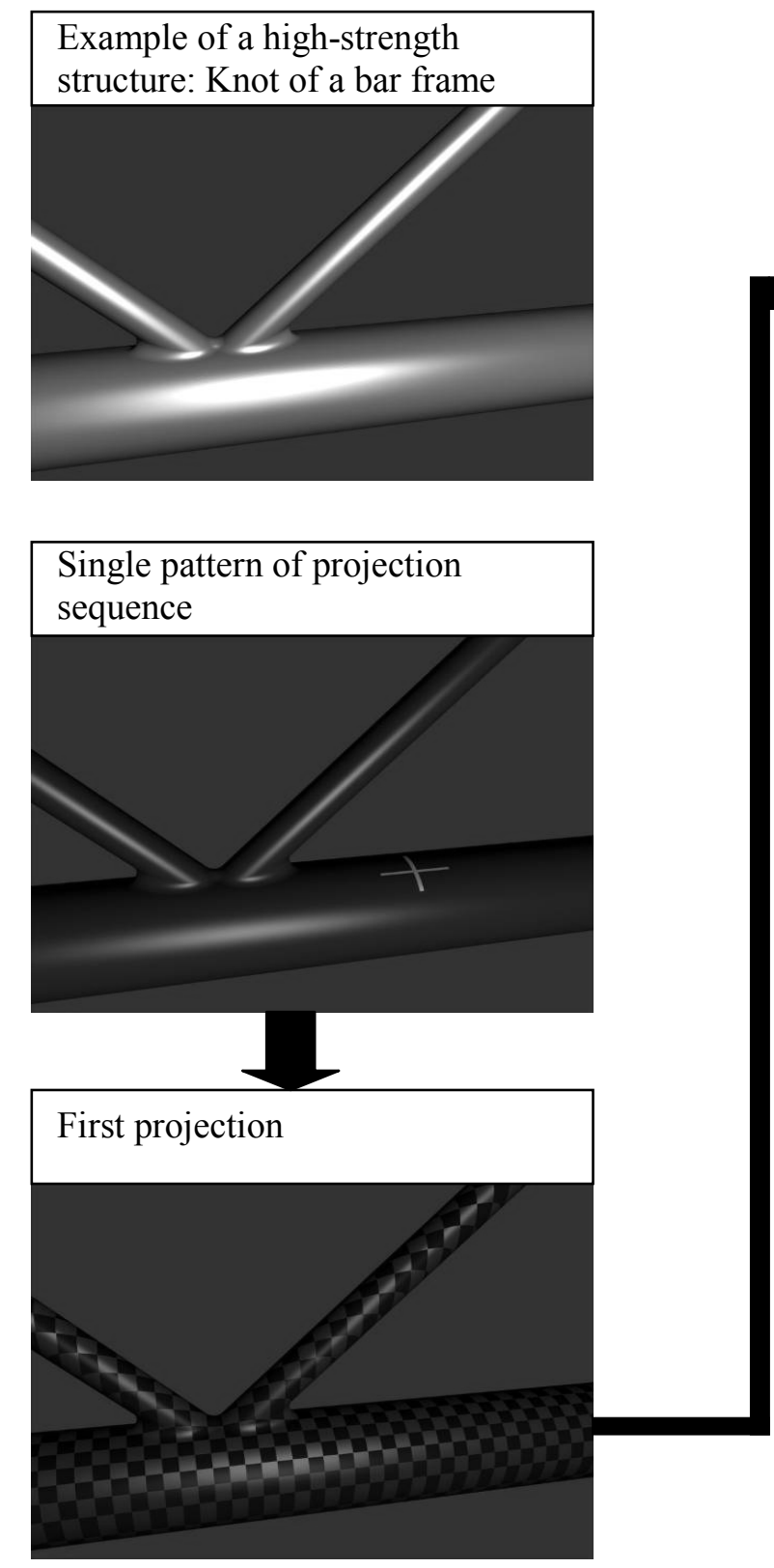
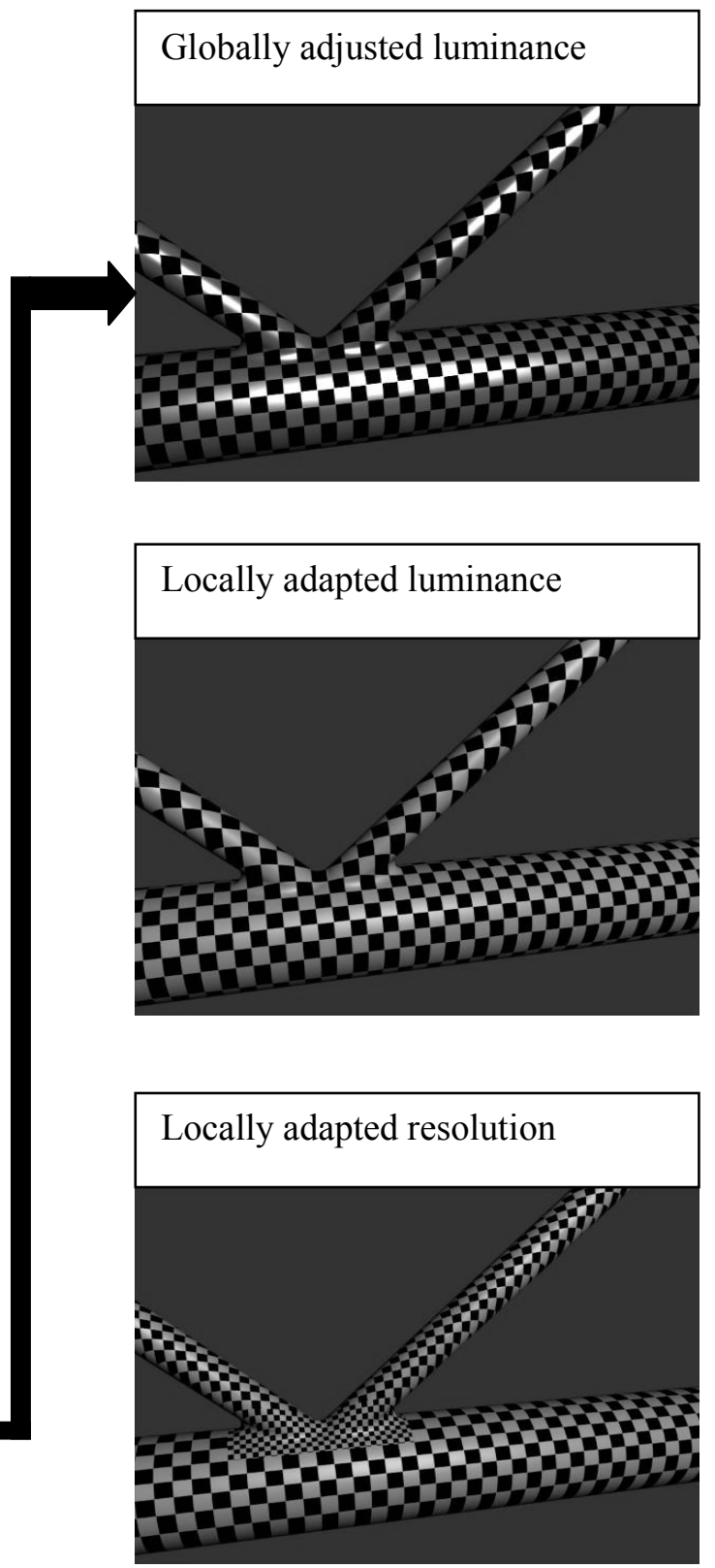

Fig. 19: Simulated two-step measurement

Additionally, there has been investigated a multi-scale projection. Instead of the sine pattern a frequency band to transmit more information with an image could be used in order to replace the time-consuming procedure of the phase-shifting. Experiments with wavelet patterns are shown in Fig. 20. Based on a basic wavelet shape a measured signal can be described as a superposition of "child wavelets" and "parent wavelets". It can be moved (translation), stretched and compressed (dilatation). The possible multi-scale transformation on the basis of wavelet transformation allows a new approach. Analogous to the Fourier transformation the discrete wavelet transformation can be applied for rough determination of the dilatation. This wavelet patterns are projected instead of sinusoidal. The local connection between the patterns in a camera and projection image can be determined by the local translation of the pattern. The filtered signal is subjected to a multi-scale decomposition, which shows the dilatations as peaks at a certain point in the transform. If dilatation is found, the camera signal in the investigated area is overlaid and the examined area is obscured 
with a wavelet signal of equivalent dilation and a local window interval. The resulting positiondependent signal can be now used to determine the translation in order to obtain a sub-pixel shift in the camera's signal as a result. In the here adopted approach the patterns of a wavelet sequence of twice used dilatation are selected. In this way an explicit translating image for the last wavelet array for each separately point can be determined from the various translations of the individual images. Fig. 20 illustrates this approach. One advantage of this technique is the possibility to attract not only the last image for the dissolution, but several, possibly even all the images, since the width of the wavelets increases the potential height resolution, but the lateral get reduced. With an appropriate model, this information can either be combined, for example for filtering outliers, or even for classification of edges. These models are the subject of current work.
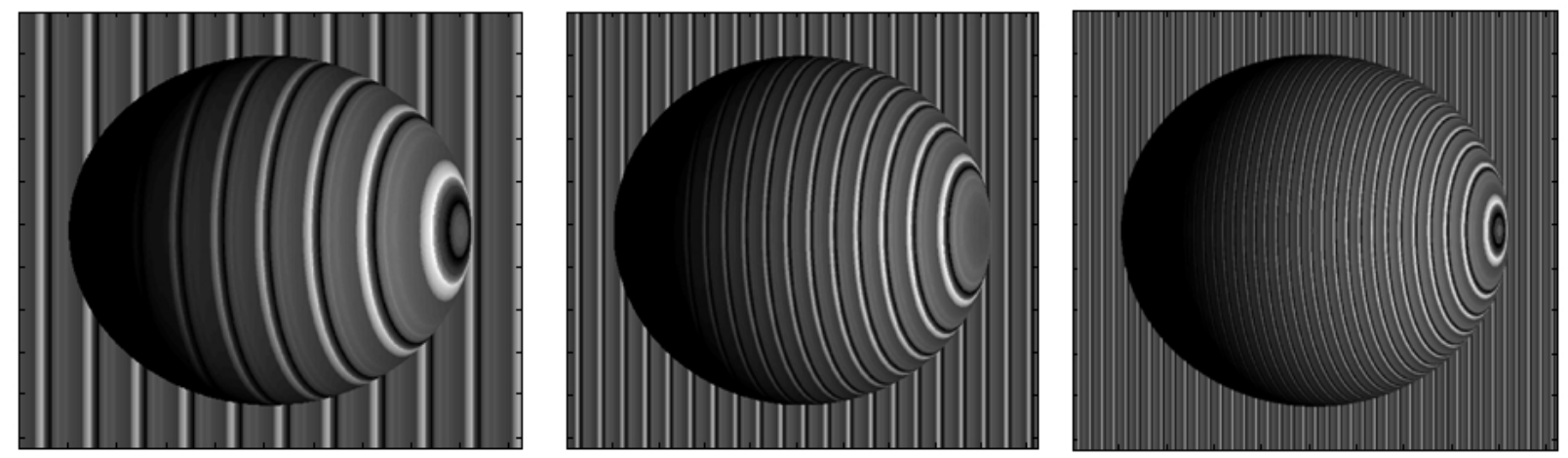

Fig. 20: Wavelet Patterns

\section{Realization of an automatic local luminance adjustment}

A key limiting factor of three-dimensional measurement through pattern projection is the brightness of the pattern in the camera image. On the one hand it varies depending on the angle of the observed surface segment relative to the camera and the projector, on the other hand on the reflection properties of the used materials. The three-dimensional geometry of the object is roughly captured in the adaptation phase. One way to improve the measurement quality is to increase the contrast on the reflection area by adjusting the projected image through reduction the projection light intensity in these problematic regions. Thereby, firstly all areas are identified in which the brightness of the reflected light is too high and overrides the camera pixels. Afterwards, a shadowed mask is calculated, which will be superimposed with the projection sequences. A second measurement cycle is performed at a higher resolution using the previously created mask. Based on the pre-measured point cloud in the first phase a surface model of the scene will be created.

Since there are a finite number of points on the surface, the intermediate values must be interpolated. One possibility is the linear interpolation with layers that are spanned through three points. Thereby a cloud of points results in a triangle mesh (Fig. 21). If there are valid neighboring points (according to Fig. 21), a triangular element will be created and its surface normal vector will be used to calculate the reflectance. 


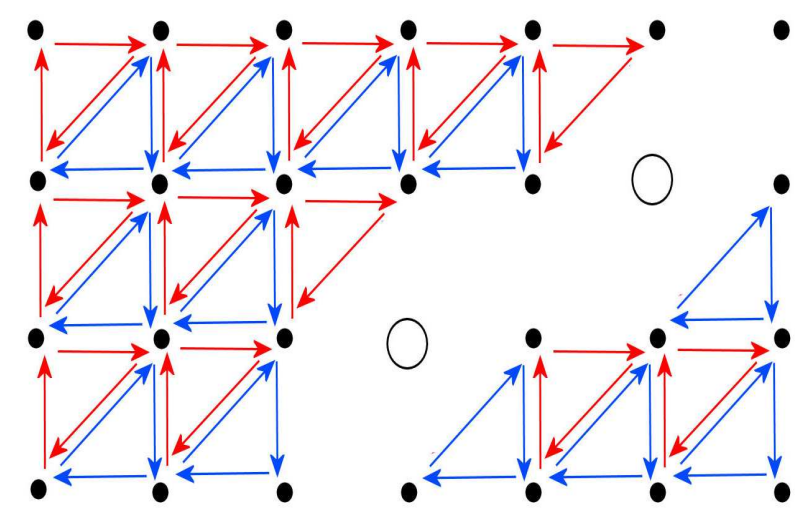

Fig. 21: Surface generation by a triangle mesh

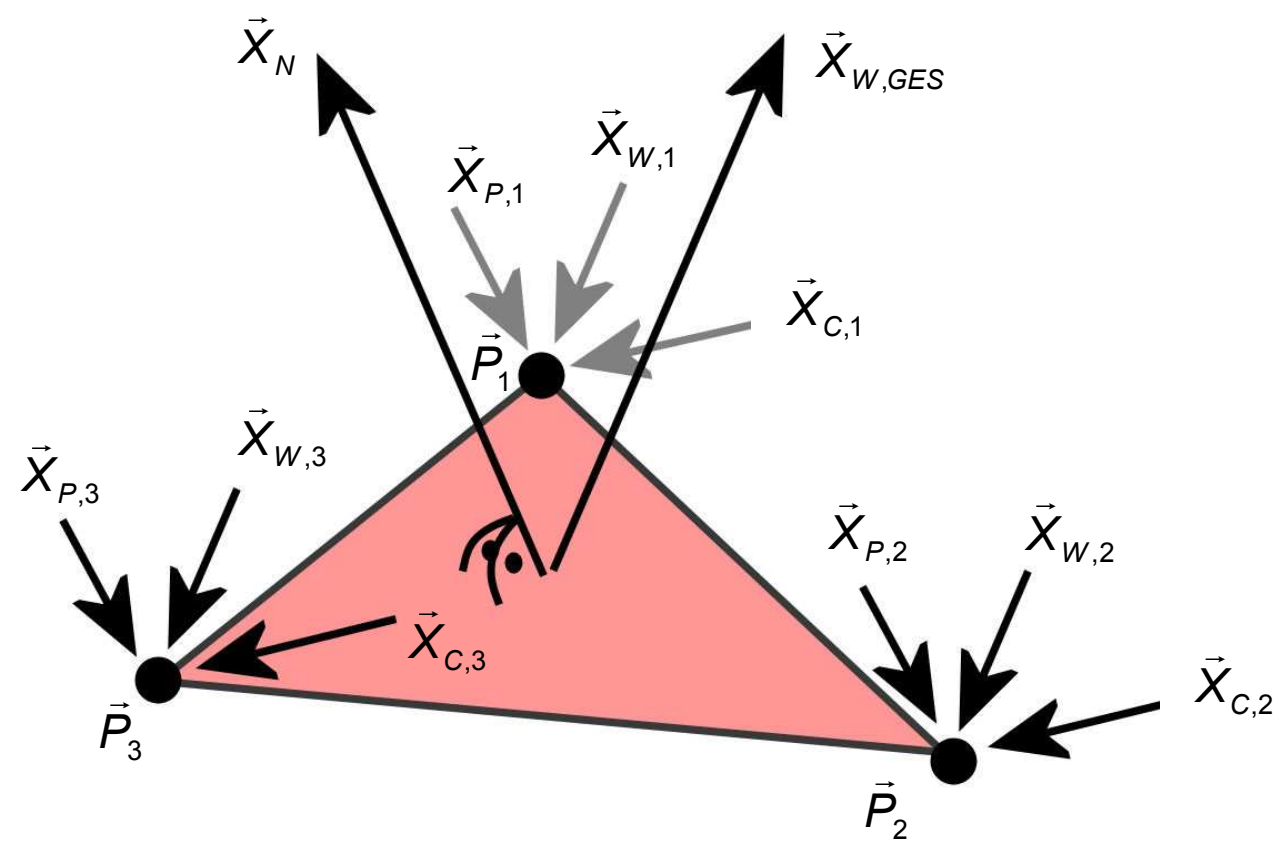

Fig. 22: Surface element

The normal vector $\vec{X}_{N}$ is perpendicular to the triangle plane and can be calculated as the normalized cross product of direction vectors $\vec{R}_{12}=\vec{P}_{2}-\vec{P}_{1}$ and $\vec{R}_{13}=\vec{P}_{3}-\vec{P}_{1}$. Also its bisector is stored for each object point $\vec{X}_{W, i}=\vec{X}_{P, i}+\vec{X}_{C, i}$, whereby $\vec{X}_{C, i}$ or $\vec{X}_{P, i}$ corresponds to the normalized vectors of camera or projector observation vector. The averaged bisecting line for the surface element can be calculated as:

$$
\vec{X}_{W, T O T}=\frac{\sum_{i=1}^{3} \vec{X}_{W, i}}{\left\|\sum_{i=1}^{3} \vec{X}_{W, i}\right\|}
$$

The scalar product of the surface normal vector $\vec{X}_{N}$ and the total bisector $\vec{X}_{W, \text { TOT }}$ yields the cosine of the angle enclosed by two vectors and can be used as a rate for the reflectance degree. If the scalar product result is close to one, the camera is positioned near to the exit angle of the directly reflected projection beam and it can be assumed that reflection effects are disturbing the 
measurement. In the next step, the critical object points are converted to the image coordinates of the projector based on the collinearity equations. Thereby, two diagonally located points define one shadowed region on the mask. In the subsequent measurement phase, the mask will be overlaid on the strip sequences as a semi-transparent object.

The approach to the luminance adjustment implemented in a simple reflection model has already shown good results with respect to the reduction of outliers. The measurement illustrated in Fig. 23a without the luminance adjustment shows significantly more outliers than the measurement in Fig. 23b with adjustment. The outliers arise from a directional reflection.

A further increase in accuracy is expected due to a more comprehensive modeling of reflections on real surfaces using the gonioreflectometer described in the next paragraph.

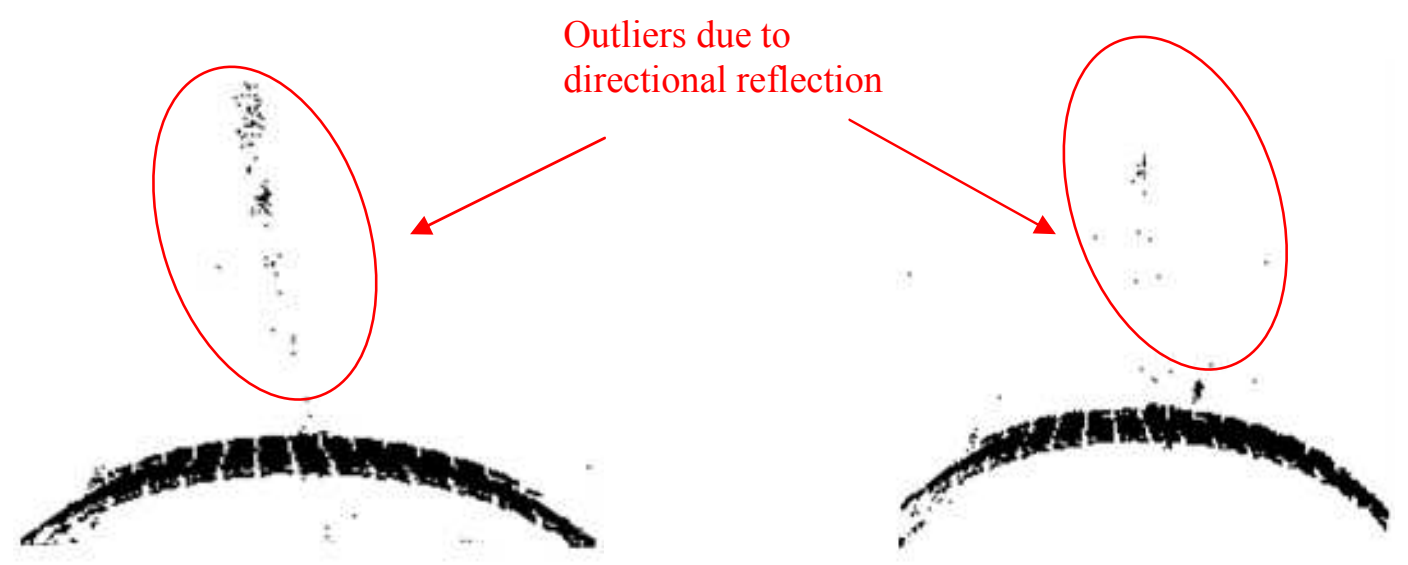

(a)

(b)

Fig. 23: Comparison of a measurement without (a) and with (b) the brightness adjustment.

\section{Gonioreflectometer}

In order to optimize the algorithm of the brightness adjustment, the reflection parameters of the surface could be adducted. Therefore, a gonioreflectometer was built. With the help of the gonioreflectometer the angle of the incident light and the angle of the light admittance by the sensor can be justified. The detection of the light power reveals conclusions about the reflection degree of the surface depending on the projector and camera angle by optical measurement.

The parameter of the reflection model will be calibrated by an experimental setup using optic fibres and rotation axes, measuring controlled reflections. The physical properties of the reflective targets are dependent on the material and surface texture. These can be included in the models. For the parameterization of the models, the angle-dependent reflectivity is investigated with the aid of the gonioreflectometer (Fig. 24). The gonioreflectometer consists of two centrally mounted rotary axes, which allows a relative positioning of the material sample and the receiving optical fiber. An arc lamp is used as the light source, which is relatively close to an ideal white light source in the spectral composition and has a high light output. The light is focused into a transmitting fiber optic cable (Fig. 25). Its output is located at a defined angle relative to the surface of the material sample. The reflected light is measured with the light unit meter. The control of rotational axes and the evaluation of the reflected light power are realized via a computer program. 

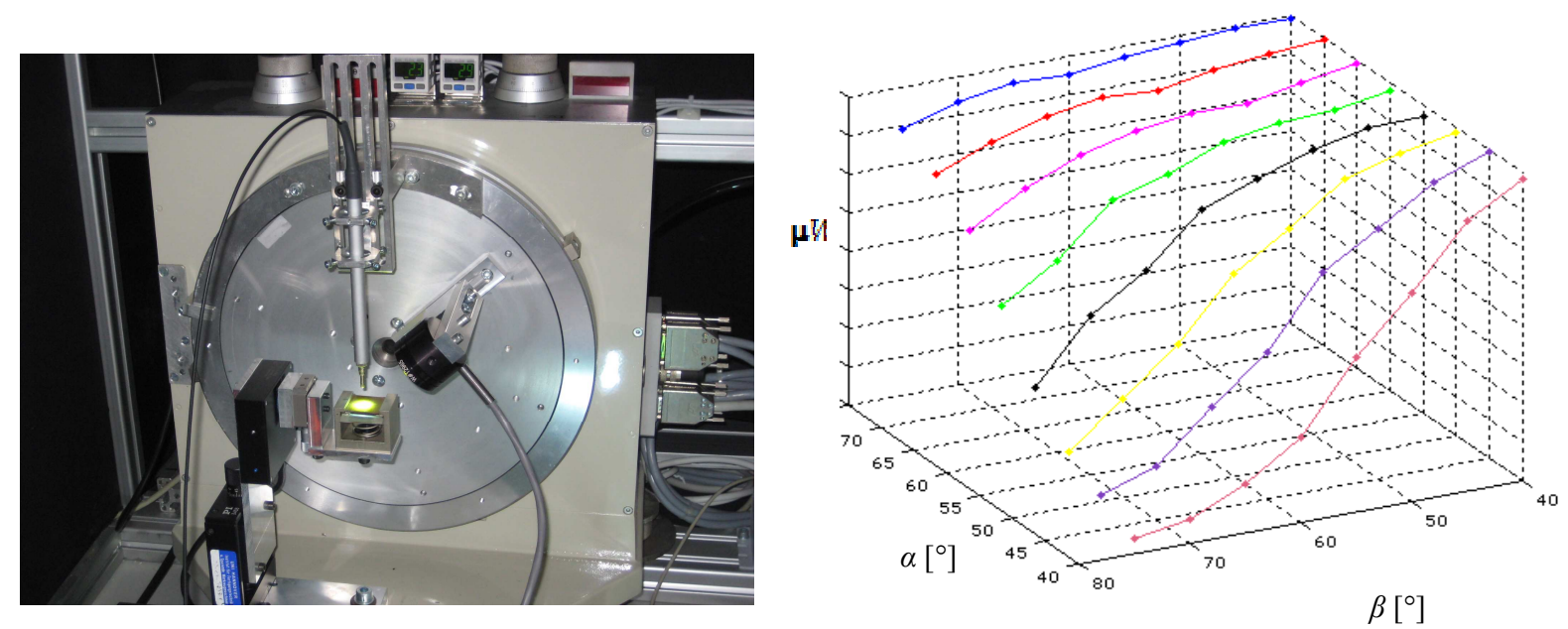

Fig. 24: Gonioreflectometer and the measurement results analysed with MatLab

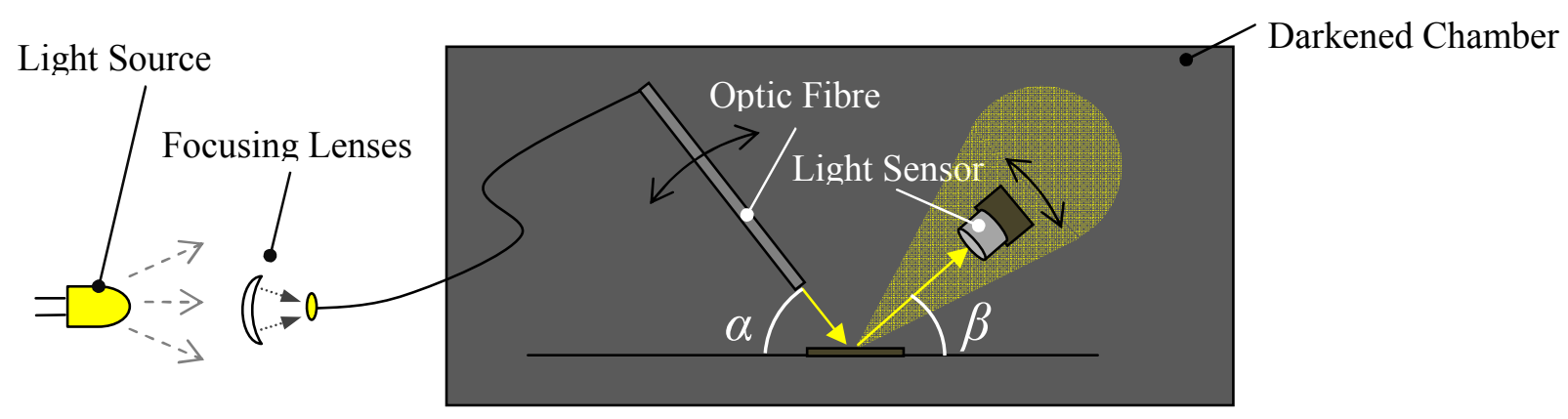

Fig. 25: Functional Principle of Gonioreflectometer

\section{The dark-field method}

It is almost impossible to detect a scratch on a reflecting surface with the naked eye looking vertically at the surface. Therefore, it is tried to select an angle to the surface, where the scratch reflects the incident light in the direction of the eye better than its surroundings. This will bring the previously invisible scratch to light up at the moment. The human brain detects the scratches because of its contrast to its surrounding surface. As a strong contrast is a very rapidly changing brightness value, a scratch can be found using the first derivative of the brightness values with respect to the position.

Scratches can be recognized in different ways by using optical measurement. One possibility is the dark-field method. In the dark field method, the examined surface is obliquely illuminated and the directly reflected light from the surface is passed to a camera. As a scratch on the surface reflects light in different directions, it is possible in a favourable camera angle to include only the light reflected from the scratch (Fig. 26). In a dark environment the scratch is identifiable by its brightness [12]. Because of the difficulty to align the camera in a suitable angle to the surface, a multidirectional lighting unit was developed at the IFW within the OptiGip-project. It makes possible to vary the otherwise rigid angle between the illumination and the object and the angle between object and camera simultaneously and automatically. The experimental setup shown in Fig. 27 was built for analysis of scratches on implants. The lighting unit consists of LEDs that are installed around the measurement object as a hemisphere in the room. The scientific results will be a base for future investigations inside the CRC 675. 


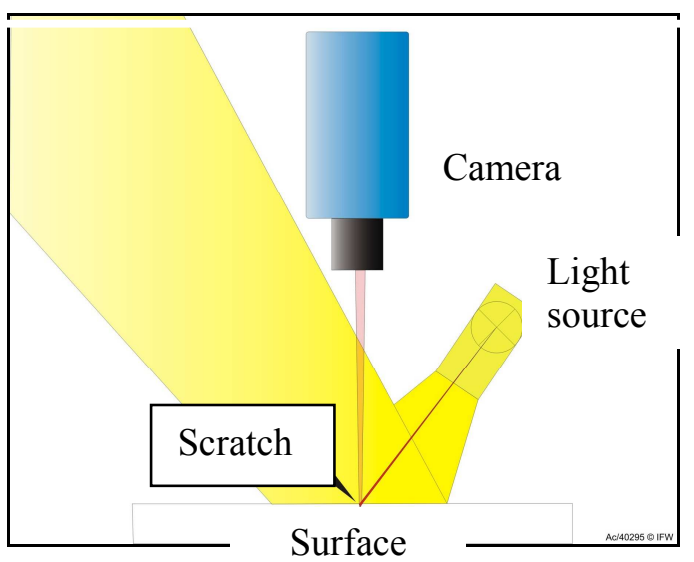

Fig. 26: Dark-field method

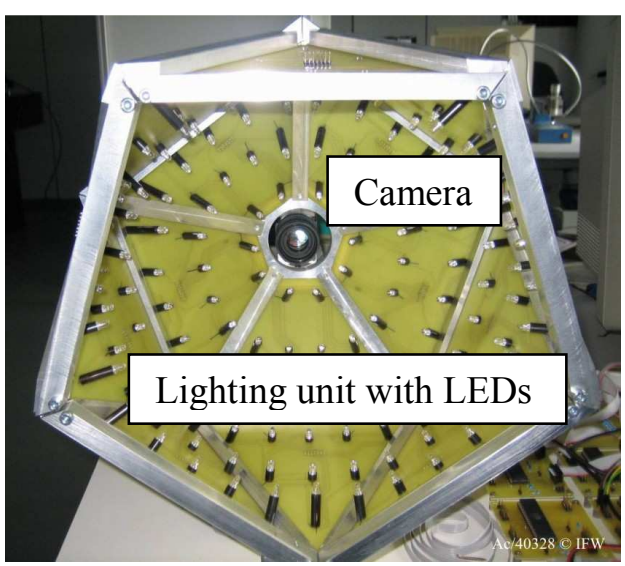

Fig. 27: Experimental setup of extended dark-field method

\section{Future research goals}

The methods of shape measurement by pattern projection with locally adapted projection parameters will be further explored. A major goal is to optimize the performance of high-strength structures by local modulation of component properties during manufacture. A key issue is the quality control of modified components with respect to their shape, mechanical properties (stiffness, strength) as well as their durability. For this reason, solutions are to be provided, allowing efficient analysis and control of novel structures. The control of load dependent structure deformations will be extended by methods for automatic identification of surface defects and cracks. The main objective is the exploration and implementation of an analytical system capable of measuring complex structures with high resolution and dynamics even under continuously changing load conditions in order to quantify the local (e.g. impact of local strain hardening, weld quality) and global (e.g. whole deformation behavior) properties. A dynamic comparison of optically recorded data on components under load and finite element (FE) simulations will become possible (Fig. 28). Furthermore, the measurement object will be placed on a turntable. The capture from different viewing angles allows the completion of the three-dimensional point cloud. Finally, the projection of adaptation and measurement configuration should be parameterized and pre-controlled with the help of optical system simulation. This offers the advantage of shorter measurement cycles on the one hand (the pre-measurement can be shortened or omitted if necessary). On the other hand, the camera-projector alignments can be calculated to take account of a mathematical optimization of accuracy and measurement capability. Thus, the fundamental question for the future research would be, in which way these additional component characteristics can be identified or examined in the quality control. 


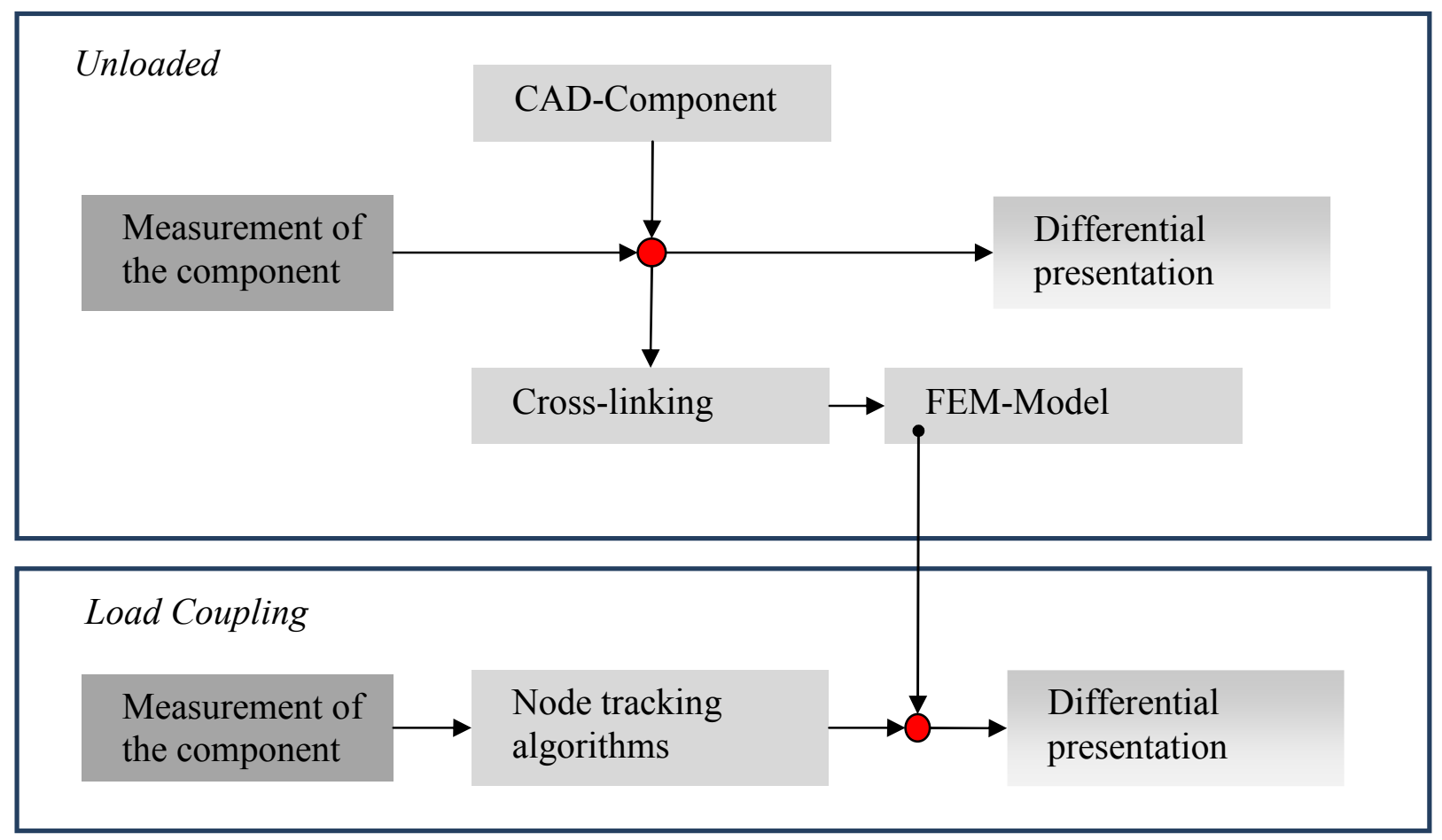

Fig. 28: Procedure of the comparative analysis between FEM and measurement

FEM-based methods for determination of real material properties will be investigated. The object will be measured under the load. With help of optically obtained deformation results and comparison with the FEM-simulated results the real material properties could be determined by iterative process of FEM-simulation, where the estimated material properties will be converged to the real ones. The principle is shown in Fig. 29.

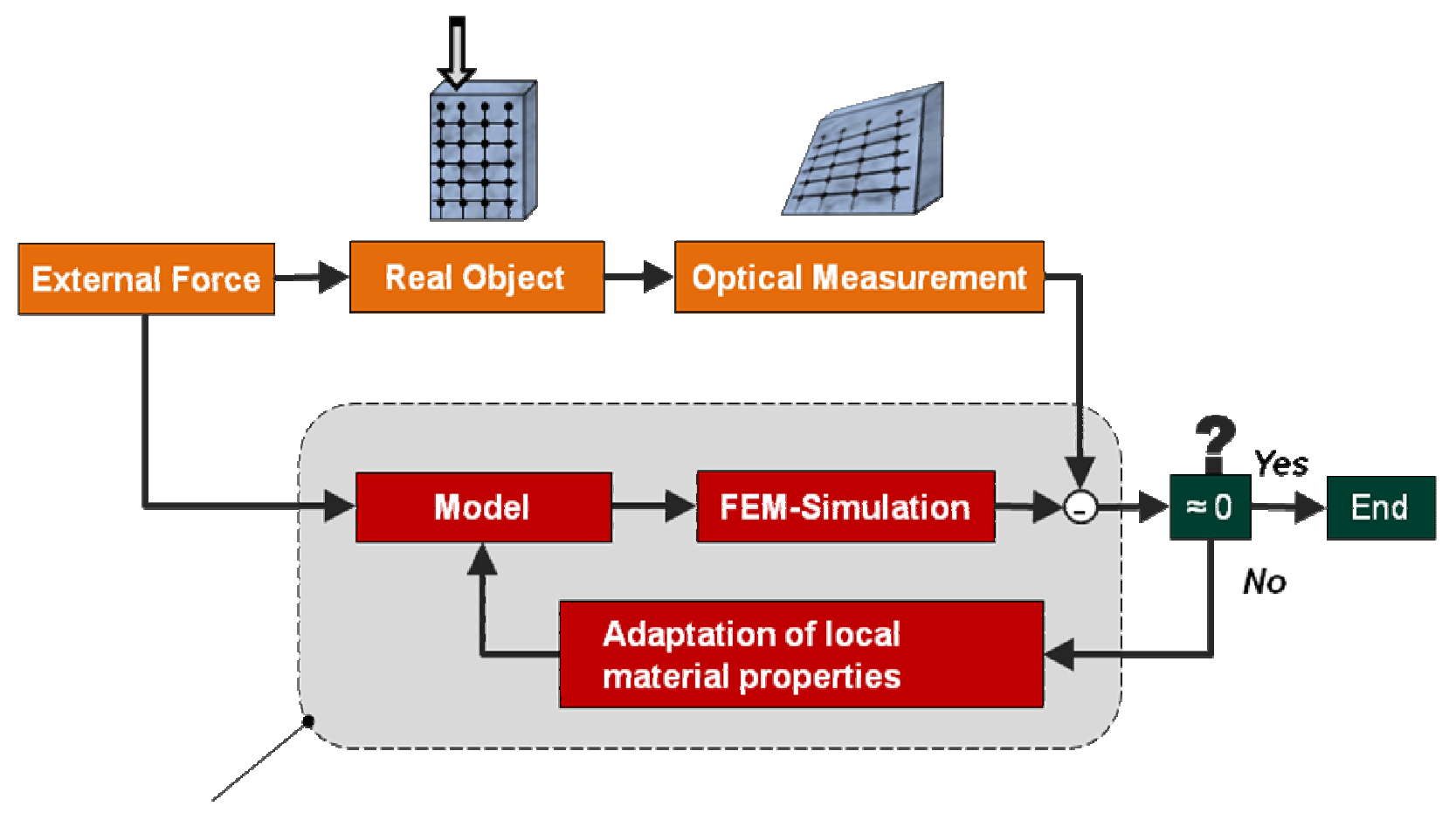

Iterative Process: convergence of estimated to real material properties

Fig. 29: FEM-based determination of local material properties 


\section{Summary}

The possibility for a optical quality control of the geometric properties of high-strength structure components has been discussed. First a variety of geometrical measurement techniques were presented and their usability for high-strength structure components examined. The one with the highest potential for the aim was identified as the method of triangulation using structured light and a "single eyed" method. The data processing problems that lead to a slow data processing speed for production purposes were discussed. Methods were described to overcome these obstacles, in order to provide the necessary robustness, measuring and evaluation speed to meet the demands of quality control in production. Therefore, a measurement approach using a locally adapted projection was introduced. The experimental setup was shown. The paper concludes with a description of how the additional information and the properties of an adapted point cloud can significantly speed up the evaluation process.

The presented research is funded by the German Research Foundation (DFG) within the project C1 of the collaborative research council 675.

\section{References}

[1] Thome Präzision, Messmaschinenkatalog, (2010)

[2] H. Steinbichler: „Holografische Interferometrie“, Messtechniken mit Lasern: Interferometrie, Holographie, Anemometrie, Speckels, Ehningen, Expert-Verlag, (1993)

[3] J.-M. Huntley: Optical shape measurement technology: Past, present, and future. Proceedings of the SPIE. The International Society for Optical Engineering, USA, Vol. 4076, pp. 162-73 (2000)

[4] T. Wolf: Streifenprojektion zur Inspektion großflächiger Bauteile in der FormteilSerienproduktion, doctoral thesis, Leibniz Universität Hannover (2003)

[5] B. Denkena, F. Berg, W. Acker: Surface Inspection System for Large Sheet Metal Parts, Erlangen (2005)

[6] H. Gärtner: Quantitative 3D-Vermessung mit Codierter Beleuchtung, Berichte aus dem Institut für Technische Optik der Universität Stuttgart, Vol. 38 (1998)

[7] R. Boecker: Problematik glänzender Oberflächen bei der 3-D-Topometrie. ABW-Workshop 5a, Technische Akademie Esslingen, (1998)

[8] B. Denkena, H.-C. Möhring, W. Acker: Erfassung und Verarbeitung von Oberflächeninformationen zur Flächenrekonstruktion. Conference Paper 3D-NordOst, Berlin (2004)

[9] B. Denkena, H.-C. Möhring, W. Acker: Reaktionsschnelle Instandsetzung dank intelligenter Digitalisierung, VDI-Z, Vol. 11/12, (2004)

[10]G. Wiora, Optische 3D-Messtechnik: Präzise Gestaltvermessung mit einem erweiterten Streifenprojektionsverfahren, Dissertation an der Ruprechts-Karls-Universität Heidelberg, (2001)

[11]W. Acker, Optical 3D Measurement of Structural Elements, Industrial Colloquium, ClausthalZellerfeld, (2007)

[12]B. Denkena; W. Acker: Optical Inspection System for Implants. Progress in Biomedical Optics and Imaging, Biophotonics and New Therapy Frontiers, Strasbourg, France, (2006) 